\title{
WestVirginiaUniversity
}

THE RESEARCH REPOSITORY @ WVU

Graduate Theses, Dissertations, and Problem Reports

2016

\section{Robust Video Stabilization and Quality Evaluation for Amateur Videos}

Usha Kalyani Alluri

Follow this and additional works at: https://researchrepository.wvu.edu/etd

\section{Recommended Citation}

Alluri, Usha Kalyani, "Robust Video Stabilization and Quality Evaluation for Amateur Videos" (2016). Graduate Theses, Dissertations, and Problem Reports. 5079.

https://researchrepository.wvu.edu/etd/5079

This Thesis is protected by copyright and/or related rights. It has been brought to you by the The Research Repository @ WVU with permission from the rights-holder(s). You are free to use this Thesis in any way that is permitted by the copyright and related rights legislation that applies to your use. For other uses you must obtain permission from the rights-holder(s) directly, unless additional rights are indicated by a Creative Commons license in the record and/ or on the work itself. This Thesis has been accepted for inclusion in WVU Graduate Theses, Dissertations, and Problem Reports collection by an authorized administrator of The Research Repository @ WVU. For more information, please contact researchrepository@mail.wvu.edu. 


\title{
Robust Video Stabilization and Quality Evaluation for Amateur Videos
}

\author{
by \\ Usha Kalyani Alluri \\ Thesis submitted to the \\ Benjamin M. Statler College of Engineering and Mineral Resources \\ at West Virginia University \\ in partial fulfilment of the requirements for the degree of \\ Master of Science \\ in \\ Electrical Engineering \\ Xin Li, Ph. D., Chair \\ Matthew C. Valenti, Ph. D. \\ Vinod K. Kulathumani, Ph. D. \\ Lane Department of Computer Science and Electrical Engineering
}

Morgantown, West Virginia

2016

Keywords: 2-D motion estimation using RANSAC and MSAC, Rapid camera panning and motion blur challenges, Total-Variation based quality evaluation metric, Objective testing. 


\section{Abstract \\ Robust Video Stabilization and Quality Evaluation for Amateur Videos \\ by}

Usha Kalyani Alluri

Amateur videos captured by consumer-level devices (e.g., mobile phones, tablets, camcorders etc.) are often shaky, undirected and difficult to watch. Video stabilization techniques attempt to improve the video quality by filtering out unwanted jittering camera motions. Currently there are two key challenges in the field of video stabilization: robustness (e.g., in the presence of sophisticated camera motion including panning and rotation) and quality evaluation. Therefore the main contributions of this thesis are two-fold.

First, we present a robust video stabilization algorithm, which post-processes shaky video data and addresses the challenges of rapid camera panning and motion blur. Our algorithm is based on a robust 2-D motion estimation method using Random Sample Consensus (RANSAC) and Mestimator Sample Consensus (MSAC). Additionally, K-Nearest Neighbor based feature replacement was developed to further improve the robustness of feature tracking. It is experimentally demonstrated that the proposed video stabilization algorithm can handle video with low feature count and motion blur.

Second, we present a Total-Variation (TV) based quality evaluation metric, which objectively quantifies the shakiness in amateur video. The proposed stabilization technique is compared against existing online video stabilization software (e.g., Deshaker and YouTube) using the newly-developed quality metric. Experimental results demonstrate that the developed algorithm is performing both subjectively and objectively at least as well as benchmark methods. 
To My Parents and Brother 


\section{Acknowledgments}

I feel very thankful and fortunate to work with Dr. Xin Li. He has been very supportive and patient all throughout my master's studies. He always encouraged my ideas, and directed my thought process towards productive learning. Our meeting discussions enhanced my problem solving skills, coding skills and inspired my perspective towards solving challenging research problem. He motivated me to work smarter and at the same time to think harder. He encouraged me to do multi-tasking and also to maintain work-life balance. I am indebted for all his help. I consider Dr. Li is my true mentor of life and I feel there is always more to learn from him.

I would like to offer my sincere thanks to Dr. Matthew Valenti, for his valuable suggestions towards this thesis. Despite his busy schedule, Dr. Valenti always responded immediately for any of my questions and encouraged me by providing valuable guidance. He has been an excellent teacher and his course has helped immensely to improve my problem solving skills. He also took great concern about my class performance and made sure that I understood the key details.

I thank Dr. Vinod Kulathumani, for accepting to serve on the committee immediately when I approached. Dr. Vinod is the one who initially identified my capabilities and highly motivated me in pursuing masters with thesis option. His teaching is very thoughtful and immensely enhanced my analytical skills. I am very thankful for his encouragement and guidance.

I am very blessed to have my brother and cousins near me to always encourage and motivate all throughout my masters studies. Without their support, I would not have been to this point. I am also thankful to my friends and well-wishers at WVU who encouraged me during my studies. Last but not the least, I remain indebted to my parents for their love and sacrifice. After all, nothing would have been possible for me without their support and encouragement. 


\section{Table of Contents}

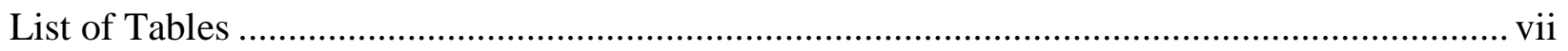

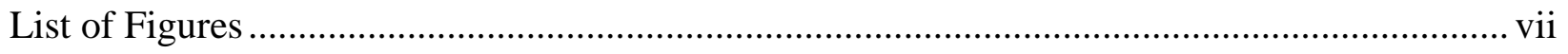

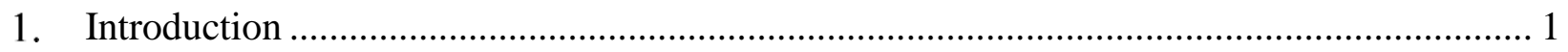

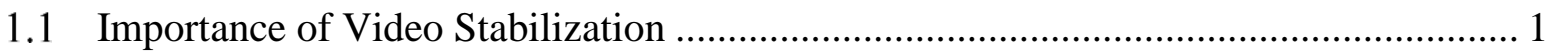

1.2 Motion Estimation in Video Stabilization.............................................................. 2

1.3 Challenges in Video Stabilization ............................................................................ 3

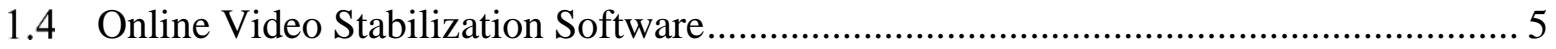

1.5 Video Data Collection and Classification ................................................................. 5

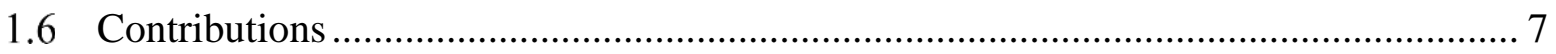

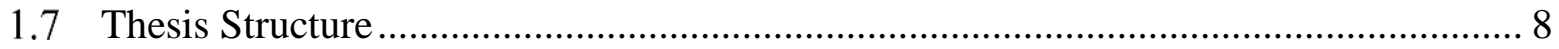

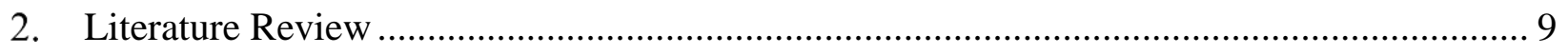

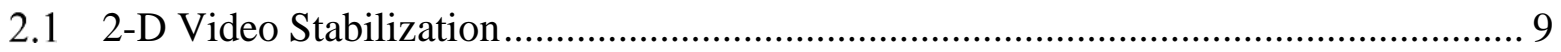

2.1.1 L1 Optimal Camera Paths Concept ………………….............................................. 9

2.1.2 Subspace Video Stabilization ............................................................................... 11

2.1.3 Bundled Camera Paths Concept .............................................................................. 14

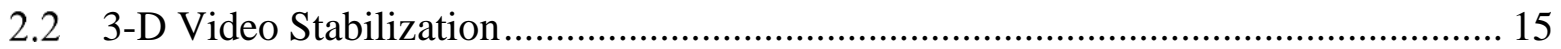

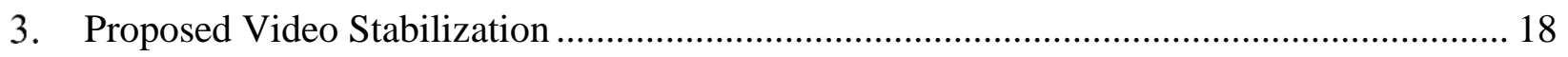

3.1 Scale Invariant Features (SIFT) and Speeded Up Robust Features (SURF) ................ 19

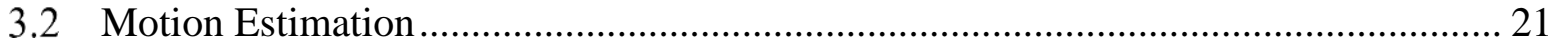

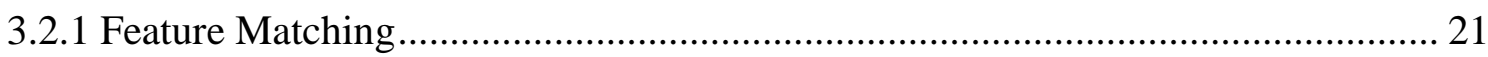

3.2.2 2-D Homography Estimation ............................................................................ 22

3.2.3 Robust Estimation using RANSAC and MSAC ………..................................... 24

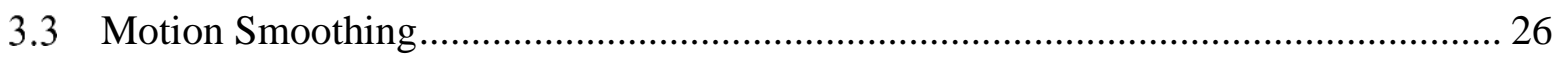

3.3.1 Path Optimization.............................................................................................. 26

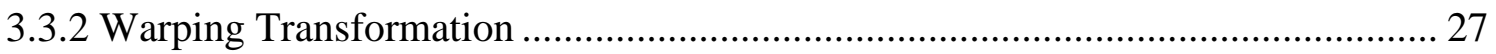

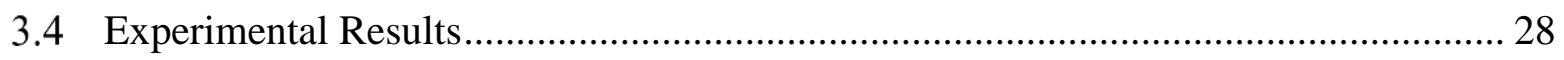

4. Proposed Quality Evaluation Metric ………………............................................... 32

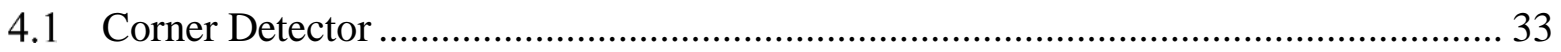




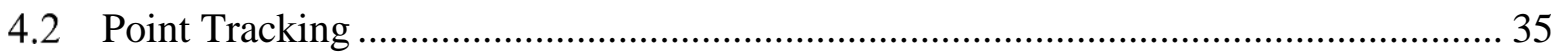

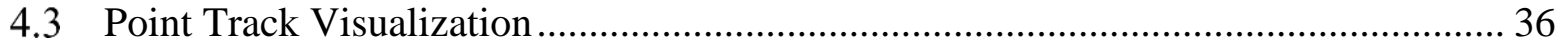

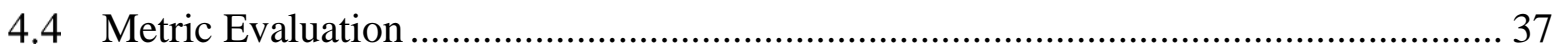

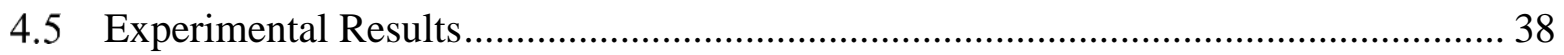

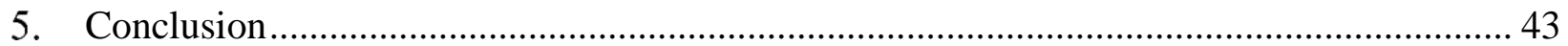

5.1 Limitations of video stabilization method .............................................................. 43

5.2 Limitations of quality evaluation metric ............................................................. 44

5.3 Future Research Directions ............................................................................... 46

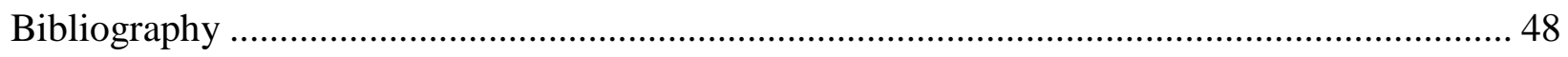




\section{List of Tables}

Table 3.1 The average run-times for the proposed algorithm for a video duration around 10 to 30 secs. 19 video sequences of each category are used in the experiment.

\section{List of Figures}

Figure 1.1 Hand-held devices and huge increase in amateur video capture............................... 1

Figure 1.2 Professional videos captured by employing expensive devices. ............................. 2

Figure 1.3 Typical video stabilization pipeline...................................................................... 3

Figure 1.4 Right: Stabilized frame using YouTube editor with shear and wobble distortion.

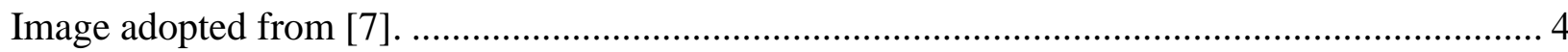

Figure 1.5 Tracked feature points are shown in color dots. Left: Tracked features without motion

blur. Right: Tracked features in the presence of motion blur. ................................................... 4

Figure 1.6 Two video frames from each of the categorized video data. (a) Simple, (b) Rotation

(c) Zooming (d) Parallax (e) Crowd (f) Running............................................................ 7

Figure 2.1 Optimal path (red) for synthetic camera path (blue) shown for various weights of the

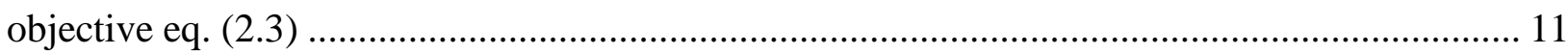

Figure 2.2 Subspace low-pass filtering. Top-left plot: A low-pass filter on two input trajectories (dashed lines) creates very different outputs (solid lines) for two similar trajectories, since their durations are different, leading to broken geometric relationships in the rendered output (a). Topright plot: if, instead, the trajectory matrix is completed using matrix factorization, the filter outputs are more similar, leading to a better rendered result (b). Images adopted from [5]......... 13 Figure 2.3 Comparison between traditional 2D stabilization (a single global camera path) and bundled camera paths stabilization model. ................................................................... 14

Figure 3.1 Proposed Video Stabilization Pipeline. .............................................................. 18 Figure 3.2 Comparative results of the proposed algorithm (b) with the Deshaker software (c) and the YouTube video editor $(\mathrm{d})$.

Figure 3.3 Top most figure shows a temporal slice from unstable video. Figure to the left shows corresponding slice in the stabilized video using our algorithm. Figure to the right is the result of Deshaker software. 
Figure 3.4 Images to the left are temporal slices of video with jittering. Images to the right are

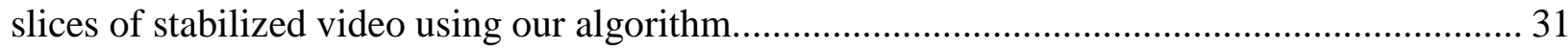

Figure 4.1 Proposed Quality Evaluation Pipeline............................................................... 32

Figure 4.2 Corner pixel has surroundings different from all its neighbors in all directions......... 33 Figure 4.312 point segment test corner detection in an image patch. The highlighted squares are the pixels used in the corner detection. The pixel at $\boldsymbol{p}$ is the centre of a candidate corner. The arc is indicated by the dashed line passes through 12 contiguous pixels which are brighter than $\boldsymbol{p}$ by

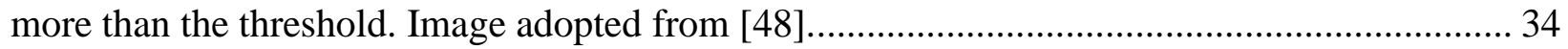

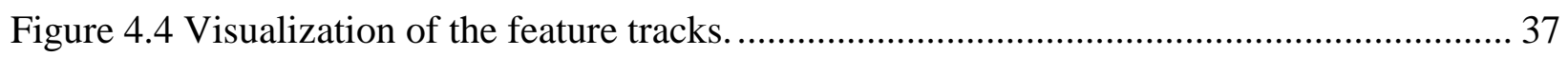

Figure 4.5 TV plot in a normal video (stable video) with walking person in the foreground..... 39 Figure 4.6 TV plot in a normal video with constant horizontal camera motion......................... 39 Figure 4.7 Jittering in the camera motion for unstable video in Simple category, compared to the stabilised videos. 40 Figure 4.8 Jittering in the camera motion for unstable video with quick rotation, compared to the stabilised videos.

Figure 4.9 Jittering in the camera motion for unstable video with crowded scene, compared to the stabilised videos. 41

Figure 4.10 Jittering in the camera motion for unstable video with rapid zooming, compared to the stabilised videos.

Figure 4.11 Jittering in the camera motion for unstable video with parallax, compared to the stabilised videos.

Figure 5.1 Wobble distortion in the stabilized video using proposed method due to sever occlusion in the foreground. 44

Figure 5.2 False camera path due to distracting foreground moving object............................. 45

Figure 5.3 False camera jittering for a video with distractive foreground. 45

Figure 5.4 Temporal slices of a stabilized video. Black patches show the missing image regions due to motion compensation. Video inpainting can be addressed as image inpainting, by interpolating each temporal slice using similar patches from same slice or neighboring slices... 47 


\section{Introduction}

\subsection{Importance of Video Stabilization}

Amateur videos that are captured using hand-held devices like cell-phones, tablets, portable camcorders etc., as shown in

Figure 1.1 contain unintentional shake of a human hand and are difficult to watch. Unlike professional videos, which are captured by employing expensive devices like tripods, camera dollies, steady cams, shoulder-support for study cams and different camera lenses, as shown in

Figure 1.2, amateur videos are unstable.
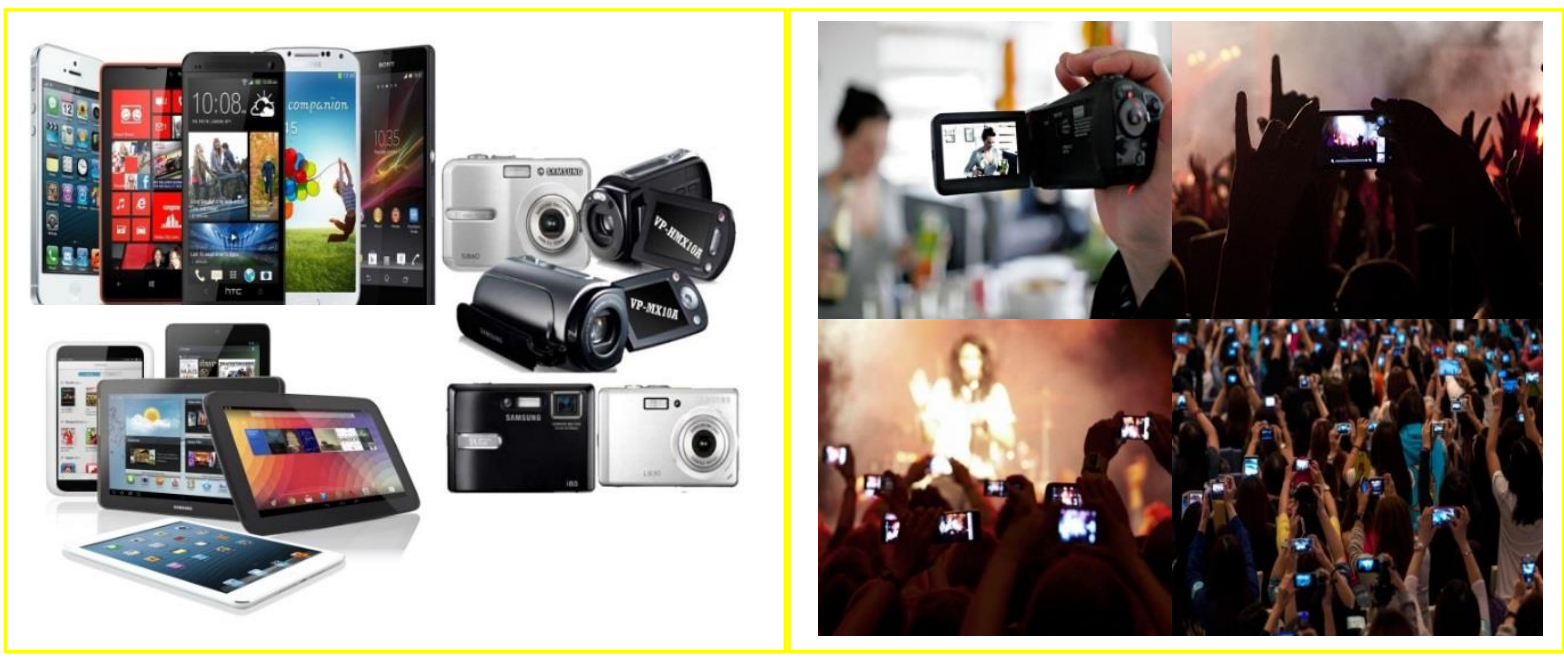

Figure 1.1 Hand-held devices and huge increase in amateur video capture.

Professional videography devices are unaffordable to the general consumers, often cannot be carried and also such hardware is impractical for many situations. So, digital video stabilization improves the video quality by processing videos captured using consumer level devices and is an important tool. 


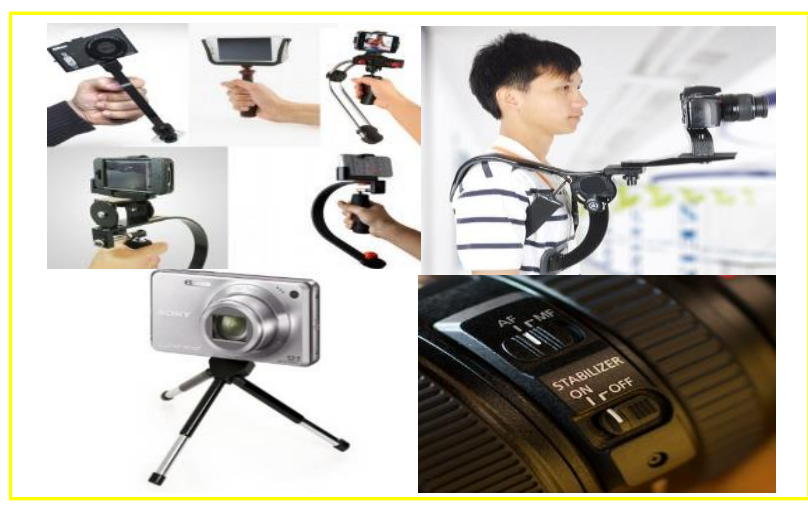

Figure 1.2 Professional videos captured by employing expensive devices.

\subsection{Motion Estimation in Video Stabilization}

Typically, video stabilization pipeline looks like shown in the Figure 1.3. Video stabilization is achieved by first estimation the shaky camera path. Conventional motion estimation approaches are two-dimensional (2-D) video stabilization [1] and three-dimensional (3-D) video stabilization $[2,3]$. 2-D motion estimation models determine the camera motion by aligning pairwise adjacent frames and estimating the transformation. 2-D motion models are robust and faster and are widely implemented in commercial software. But the 2-D motion model cannot handle the parallax caused by depth changes in 3-D camera motion, so the amount of stabilization it can provide is limited. In contrast to conventional 2-D video stabilization techniques, conventional 3$\mathrm{D}$ video stabilization techniques may perform much stronger stabilization. 3-D models can handle parallax in principle and can perform much stronger stabilization. 3-D based methods reconstruct the 3-D camera poses using structure from motion (SFM) algorithms [4]. But the 3-D motion model is too complex and less robust to compute. 3-D reconstruction is also less robust to feature tracking failure, motion blur and camera zooming. In the following work, the challenges in video stabilization and devised algorithm that has the benefits of 2-D and 3-D video stabilization are discussed.

There is another motion model called as 2.5-D inter-frame motion model [8], that can work in situations where significant depth changes are present and the camera has both rotational and translational movements. 2.5-D models relax the requirement of full 3-D reconstruction to some partial 3-D information such as the epipolar geometry [9]. Though the 2.5-D model has a tempting 
feature of introducing partial depth information into the 2-D model, the algorithm is practically difficult to realize, and so it is not detailed in this thesis.

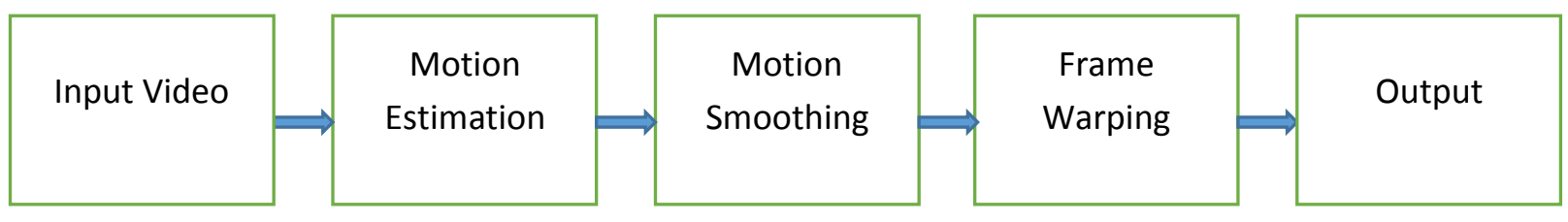

Figure 1.3 Typical video stabilization pipeline.

\subsection{Challenges in Video Stabilization}

There were many video stabilization techniques developed in recent years and stabilization algorithms have been improved dramatically. Some algorithms are successfully embedded into commercial software, for example the Warp stabilizer in Adobe After Effects was built on subspace video stabilization method [5] and YouTube stabilizer was developed from homography mixture models [6]. Despite these successes, there are still some challenges in the video stabilization techniques and few of them are discussed below.

Simple motion models are invalid when there are large depth changes in the scene, and which is very common in consumer videos. The best fitting homography cannot describe all the motions of the scene, resulting the distortion and wobbling as shown in Figure 1.4.

Rapid camera panning and large dynamic occlusion are other challenge for video stabilization. Video stabilization methods rely on tracking features points through the frames. However, in the presence of rapid camera panning and larger size moving objects, the number of tracked feature points and the length of feature tracks drop significantly, which makes feature tracking based video stabilization fragile. 

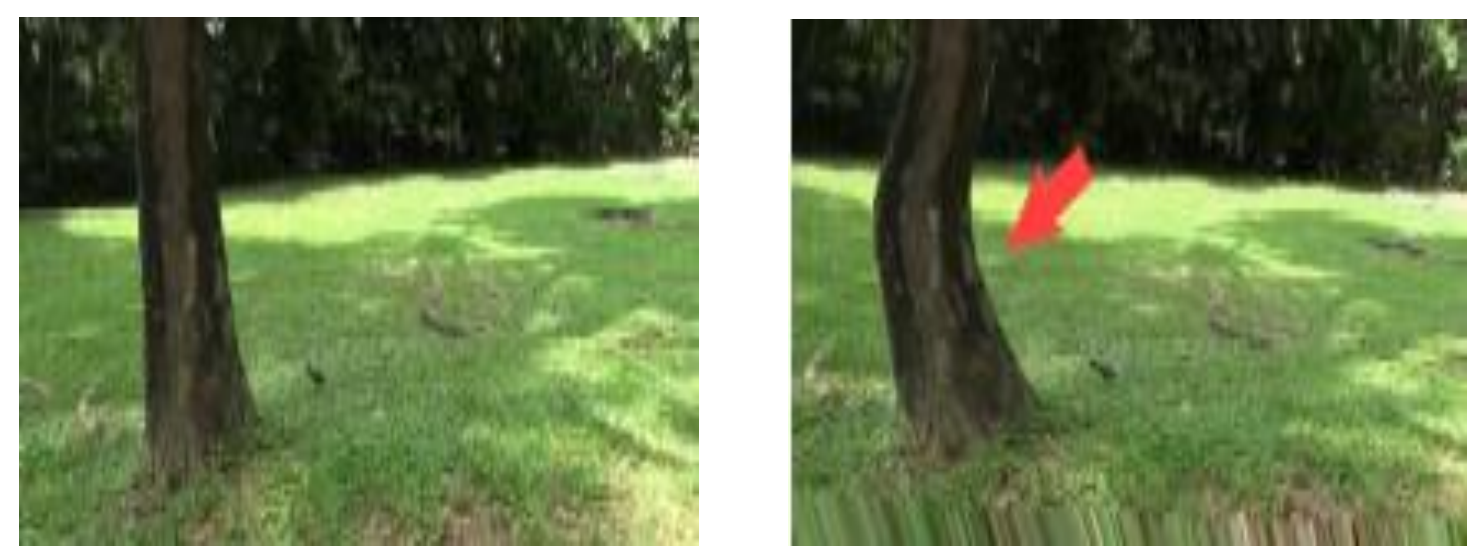

Figure 1.4 Right: Stabilized frame using YouTube editor with shear and wobble distortion. Image adopted from [7].

Motion blur can also result in losing feature trajectory over blurry frames as shown in Figure 1.5. In some cases when there is larger camera shake, feature extraction completely fails. So, motion bur is important to handle for a robust video stabilization algorithm.
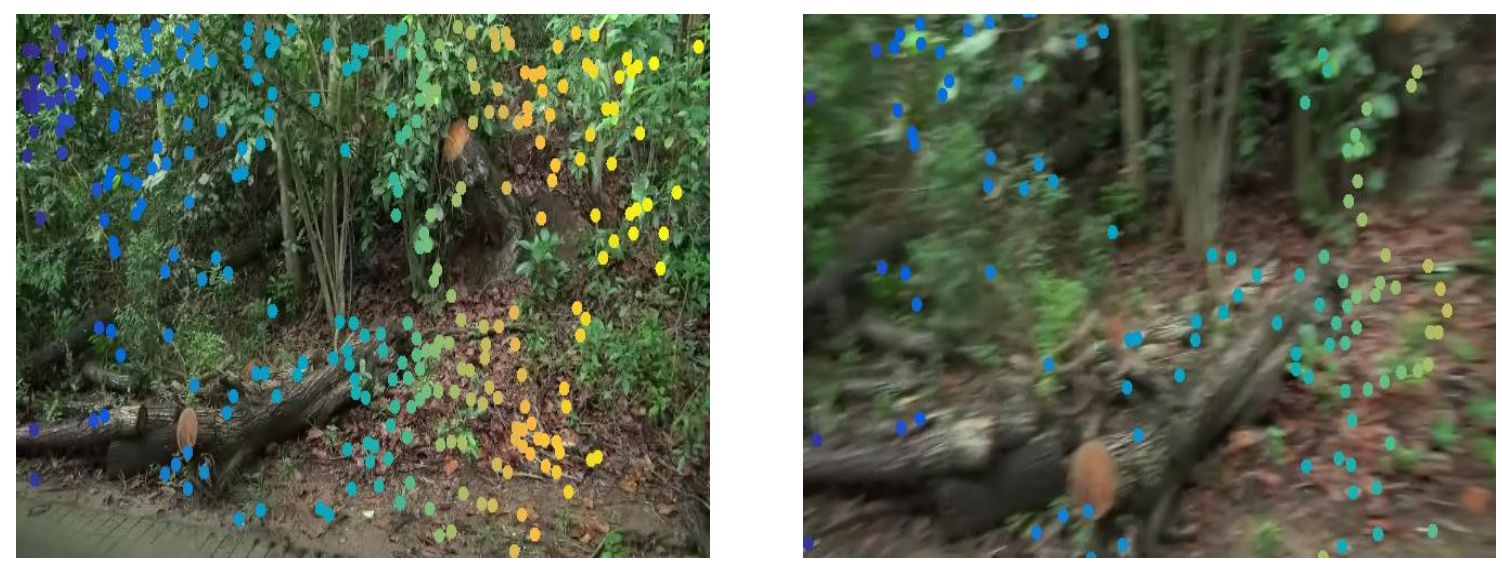

Figure 1.5 Tracked feature points are shown in color dots. Left: Tracked features without motion blur. Right: Tracked features in the presence of motion blur. 


\subsection{Online Video Stabilization Software}

Deshaker ${ }^{1}$ is one of the free online available video stabilization software. It is an added filter for VirtualDub (VirtualDub is a video capture/processing utility for 32-bit and 64-bit Windows platforms). It eliminates camera shakiness and makes panning, rotation and zooming smoother. Installation setup and using this software is not obvious. Also, for high foreground motion, the output results from this software suffer from high-frequency vibrations and visually worse than the input.

YouTube Video Editor ${ }^{2}$ has a feature to stabilize the handheld videos each-time when we upload a video onto YouTube. It is strictly an online service and works well only for simple videos that doesn't suffer complex camera shakes. The camera path estimation of the stabilizer is unreliable when video data has low feature count, excessive blur during extremely fast motions or lack of rigid objects in the scene [16].

Windows Movie Maker (2012 version) in Windows 8 has added a new Video Stabilization button, with options for anti-shake and wobble corrections. Microsoft claims that the feature takes advantage of Windows 8's graphics hardware acceleration to speed up the task of stabilization. But, this feature resulted to produce an output video that only compensates little bit of wobbling and shaking and also the output video is not good to watch.

Video stabilization feature is also available in other software's like Warp Stabilizer in Adobe After Effects, iMovie stabilization, etc., which come along when the software is bought so they are not discussed in this work.

\subsection{Video Data Collection and Classification}

Video dataset that has been available publicly from various video stabilization publications is collected and used in this work. Most of the video data is collected from [7] and [5]. [7] have nicely categorized the data based on camera motion and scene type as, (I) simple, (II) rotation, (III) zooming, (IV) parallax, (V) crowd and (VI) running. For the understanding of reader, two frames

${ }^{1}$ http://www.guthspot.se/video/deshaker.htm

${ }^{2}$ https://www.youtube.com/editor 
from each data category are shown in Figure 1.6. Some of the video data is also captured using iPhone 5 and Samsung Galaxy S4 around our University.

(a)
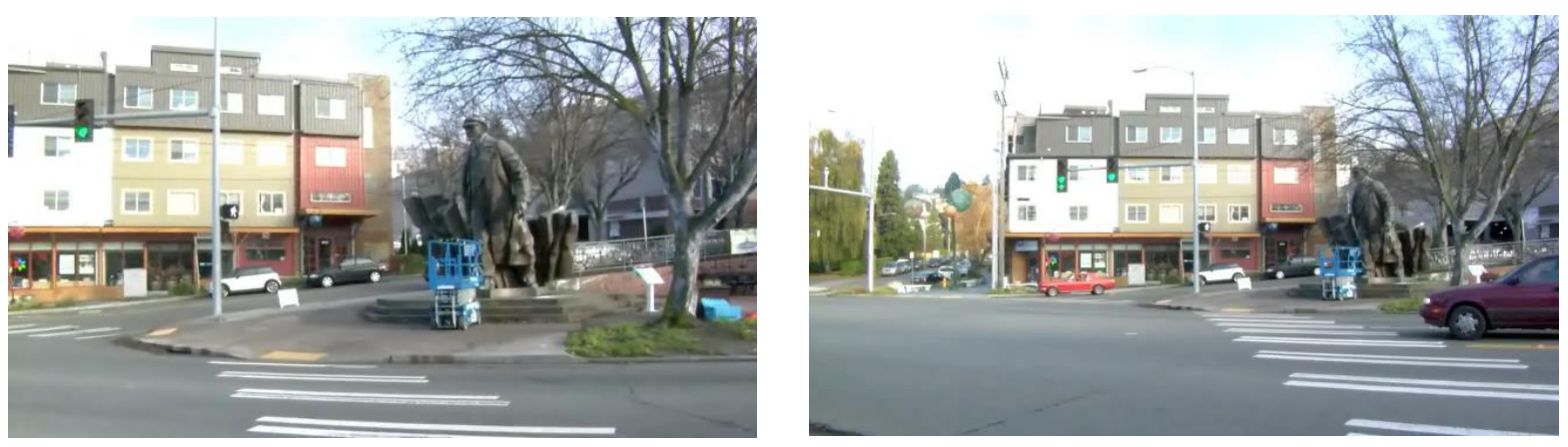

(b)
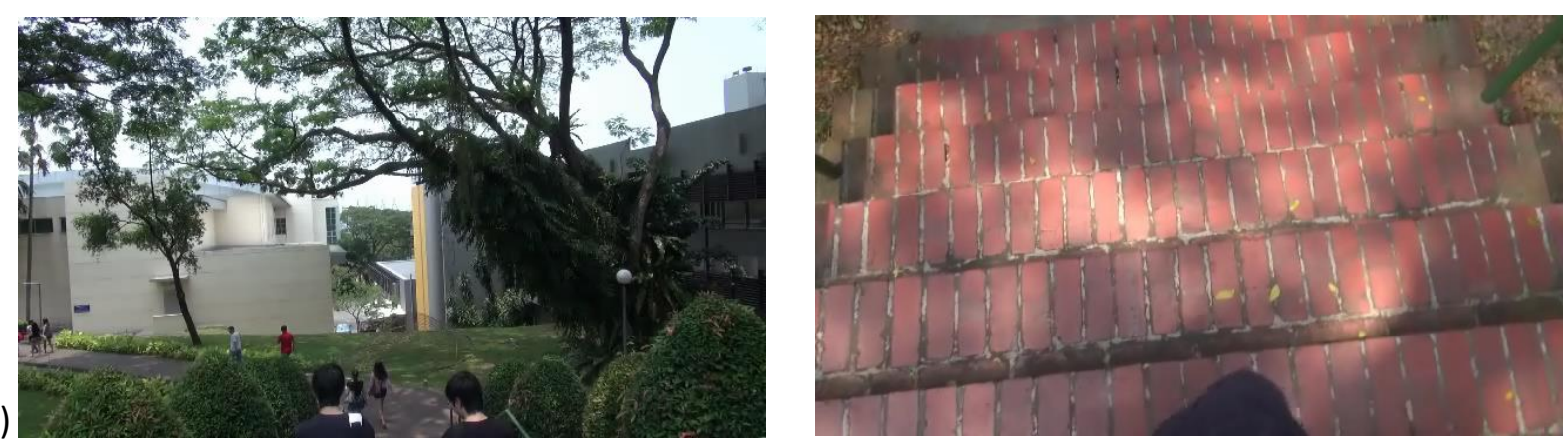

(c)
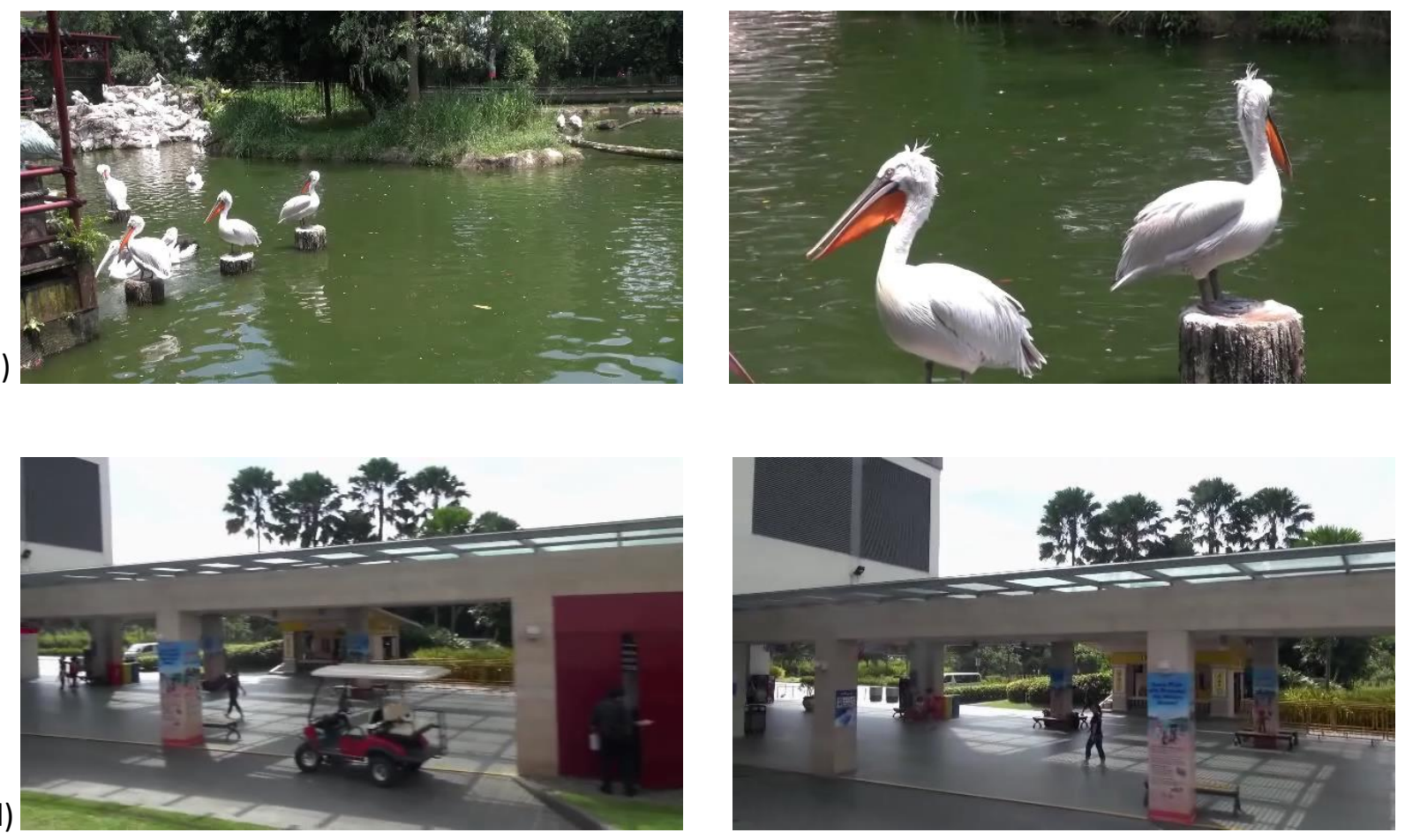
(e)
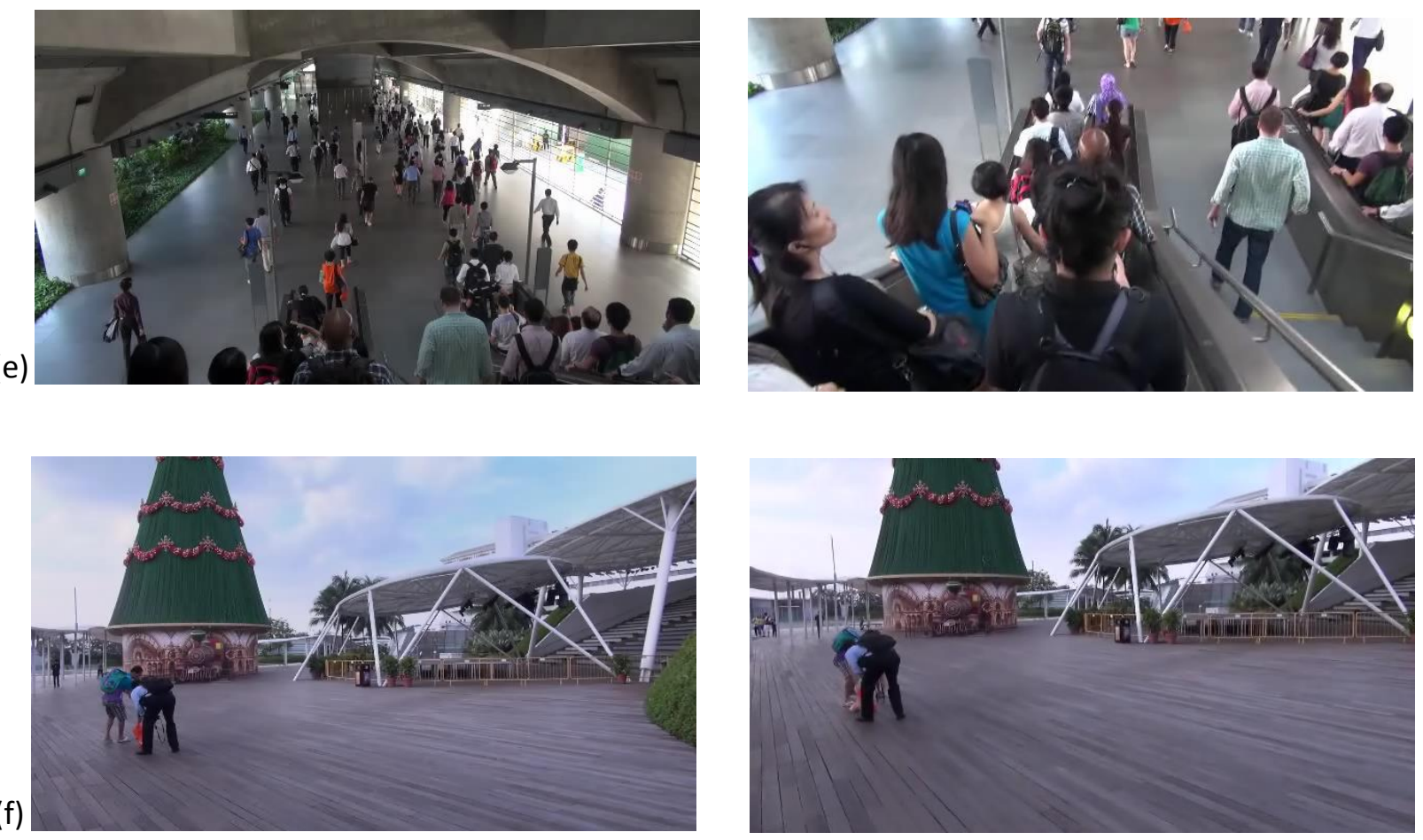

Figure 1.6 Two video frames from each of the categorized video data. (a) Simple, (b) Rotation (c) Zooming (d) Parallax (e) Crowd (f) Running.

\subsection{Contributions}

A robust video stabilization algorithm should be capable of handling as many stabilization challenges as possible with optimized implementation and less computational complexity. The homography based methods are less complex but, feature point detection and tracking is invalid when there is rapid camera panning, large dynamic occlusion and motion blur.

Contribution of this work is to address these challenges by detecting and separately handling the frames that suffer rapid camera panning, large dynamic occlusion and motion blur. Rest of the frames are normally handled using homography estimation to keep the algorithm optimized and less complex.

Proposed total-variation (TV) based qualitative evaluation helps visualize and compare the jittering in the camera path before and after stabilization, which benefits on accurately estimating 
the robustness of the developed algorithm. Quality evaluation also benefited in evaluating other video stabilization software. A robust video stabilization algorithm must also address excessive cropping after motion compensation. At the end of this thesis, we propose a temporal based video inpainting idea which can help to reduce cropping and fully reconstruct the video frames, but it needs to be computationally improved in the future.

\subsection{Thesis Structure}

Chapter 2 briefly provides a comprehensive literature review on the previous video stabilization algorithms. Chapter 3 presents the proposed video stabilization algorithm with experimental results showing the average run-time of our method compared to Deshaker software. Chapter 4 presents the quality evaluation matric with extensive experimental results. Chapter 5 concludes the work by presenting the limitations of this work and future research scope along this direction. 


\section{Literature Review}

The two main video stabilization models, 2-D and 3-D are discussed in this chapter.

\subsection{2-D Video Stabilization}

2-D motion models estimate 2-D transformations between consecutive video frames. Basically, homography or affine transformation is used to represent the camera motion. These transformations are concatenated to estimate the 2-D camera path. Then the estimated camera path is smoothed to obtain the stabilized video. Strictly speaking, a homography model is only valid when the scene is planer or the camera undergoes purely rotational motion [4]. Prior stabilization techniques such as $[1,10,11]$ estimated affine transformations or homographies between consecutive frames and applied low pass filtering to reduce high frequency camera jitters [12].

Chen et al. [13] fits polynomial curves to camera trajectories to suppress low frequency camera shakes. Gleicher and Liu [14] further broke camera trajectories into segments and fitted smooth motion to each of them for better camera motion. Grundmann et al. [16] applied cinematography rules [15] and represented camera motion by a combination of constant, linear or parabolic motion. As this technique has been robust and follows cinematography rules, it has been integrated into Google YouTube and works well on many casual online videos.

\subsubsection{L1 Optimal Camera Paths Concept}

From a cinematographic standpoint, the most pleasant viewing experience is conveyed by the use of either static cameras, panning ones mounted on tripods or cameras placed onto a dolly. To mimic professional footage, Grundmann et al. [16] has optimized the paths to be composed of the following path segments:

- A constant path, representing a static camera, i.e. $D P(t)=0, D$ being the differential operator and $P(t)$ is the computed camera path.

- A path of constant velocity, representing a panning or a dolly shot, i.e. $D^{2} P(t)=0$.

- A path of constant acceleration, representing the ease-in and out transition between static and panning cameras, i.e. $D^{3} P(t)=0$. 
To obtain the optimal path, Grundmann et al. [16] formulated the problem as a constrained L1 minimization. L1 optimization has the property that the resulting solution is sparse, i.e. it will attempt to satisfy many of the above properties along the path exactly. Also L1 optimized path is only composed of segments resembling a static camera, (uniform) linear motion, and constant acceleration [16].

Grundmann et al. [16] used linear programming to solve for optimal camera path, which is as follow. If the video is considered to be a sequence of images $I_{1}, I_{2}, \ldots I_{n}$, where each frame pair $\left(I_{t-1}, I_{t}\right)$ is associated with a linear motion model $F_{t}$. The camera path $C_{t}$ is defined as:

$$
C_{t+1}=C_{t} F_{t+1} \Rightarrow C_{t}=F_{1} F_{2} \ldots F_{t}
$$

Given the original path $C_{t}$, the desired optimal path is denoted as:

$$
P_{t}=C_{t} B_{t}
$$

where $B_{t}=C_{t}^{-1} P_{t}$ is the update transform which when applied to original path, yields the optimal path. The optimization finds the optimal stable path by minimizing the objective function (2.3) using linear programming:

$$
O(P)=w_{1}|D(P)|_{1}+w_{2}\left|D^{2}(P)\right|_{1}+w_{3}\left|D^{3}(P)\right|_{1}
$$

weights w1 - w3 in the objective eq. (2.3) are chosen such that, w3 to be an order of magnitude larger than both w1 and w2 to eliminate jerks and to convey a more pleasant viewing experience Figure 2.1 . 

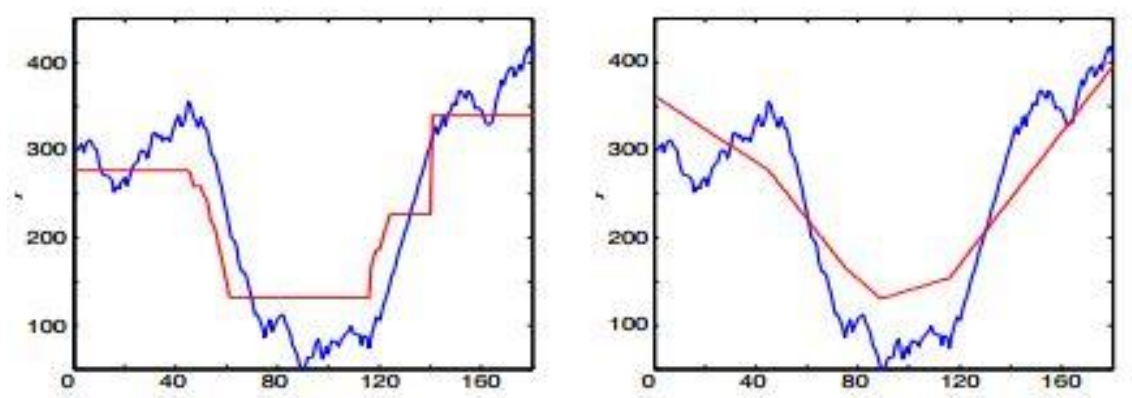

(a) $w_{1}=1, w_{2}=w_{3}=0$

(b) $w_{2}=1, w_{1}=w_{3}=0$
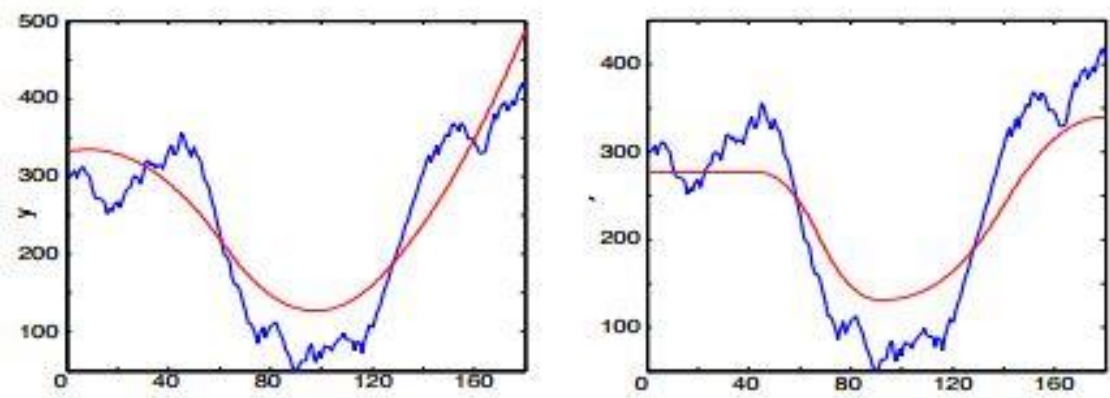

(c) $w_{3}=1, w_{1}=w_{2}=0$

(d) $w_{1}=10, w_{2}=1, w_{3}=100$

Figure 2.1 Optimal path (red) for synthetic camera path (blue) shown for various weights of the objective eq. (2.3)

\subsubsection{Subspace Video Stabilization}

Recently, Liu et al. 2011 [5] introduced a technique that imposes subspace constraints [21] on feature trajectories when computing the smooth paths. However, this method requires long feature tracks over multiple frames.

Subspace approach to video stabilization consists of four steps.

- First, standard 2D point tracking is used and assemble the 2D trajectories of sparse scene points into an incomplete trajectory matrix.

- Second, perform moving factorization to efficiently find a time-varying subspace approximation to the input motion that locally represents the trajectories as the product of basis vectors called as eigen-trajectories and a coefficient matrix that describes each feature as a linear combination of these eigen-trajectories. 
- Third, perform motion planning (or smoothing) on the eigen trajectories, effectively smoothing the input motion while respecting the low-rank relationship of the motion of points in the scene.

- Fourth, the eigen-trajectories are re-multiplied with the original coefficient matrix to yield a set of smoothed output trajectories that can be passed to a rendering solution, such as content-preserving warps [2], to create a final result.

The set of $\mathrm{N}$ input feature trajectories across $\mathrm{F}$ frames whose $i-t h$ trajectory is $\left\{\left(x_{t}^{i}, y_{t}^{i}\right.\right.$ )$\}$, where $\left(x_{t}^{i}, y_{t}^{i}\right)$ are coordinates at frame t. These trajectories can be concatenated into a trajectory matrix $\mathrm{M}$ :

$$
M_{2 N \times F}=\left[\begin{array}{lllc}
x_{1}^{1} & x_{2}^{1} & & x_{F}^{1} \\
y_{1}^{1} & y_{2}^{1} & & y_{F}^{1} \\
& \vdots & & \vdots \\
x_{1}^{N} & x_{2}^{N} & & x_{F}^{N} \\
y_{1}^{N} & y_{2}^{N} & & y_{F}^{N}
\end{array}\right]
$$

A low-pass filter is not directly applied to this matrix, if so distortion would happen, as independently smoothing feature trajectories breakdown the relationship between points. Figure 2.2 left shows such an example. To maintain this relationship during the smoothing, a subspace constraint is proposed, Figure 2.2 right shows an example. In general, motion trajectories from a perspective camera will lie on a non-linear manifold $[22,23]$. It is possible to approximate the manifold locally with a linear subspace. Irani [24] showed that the trajectory matrix should have at most rank 9. This low-rank constraint implied that the trajectory matrix $\mathrm{M}$ can be factored into the product of two low-rank matrices:

$$
M_{2 n \times k} \approx W \odot\left(C_{2 n \times r} E_{r \times k}\right)
$$

where $W$ is a binary mask matrix indicating missing data, and $\odot$ means component-wise multiplication. $E$ is the eigen-trajectories and $C$ contains the coefficient for the linear combination. If we apply a smooth operation $\mathrm{K}$, it can be further derived as:

$$
\widehat{M}=W \odot(C E) K=W \odot C(E K)=W \odot C \widehat{E}
$$


which means we first filtering the eigen-trajectories $E$ to obtain $\hat{E}$, and then obtain a new sub matrix $\widehat{M}_{2 n \times k}$ by multiplying $\hat{E}$ with the original coefficient matrix $C$. Output frames can be obtained by content-preserving warp guided by the control points in $M$ and $\widehat{M}$.
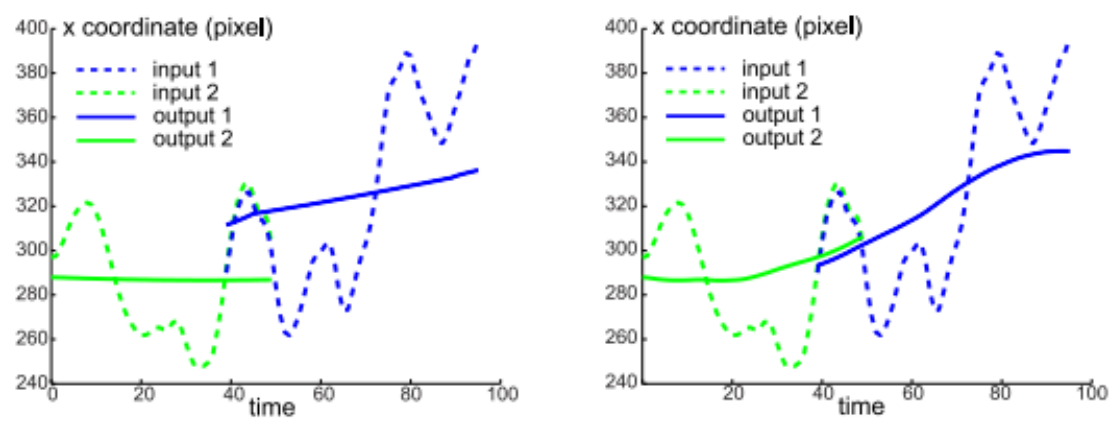

(a)

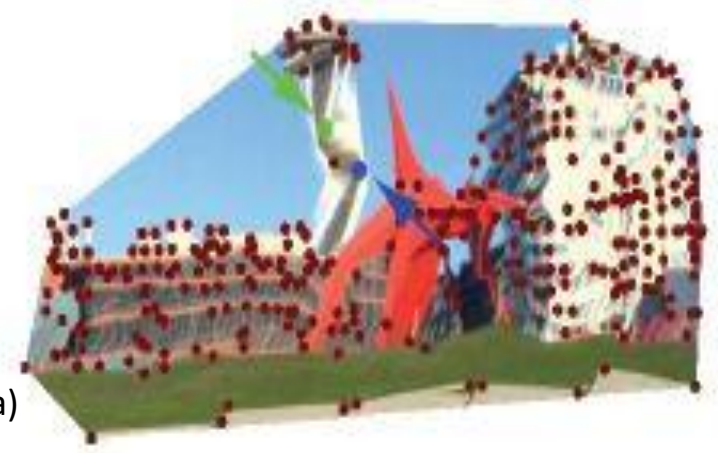

(b)

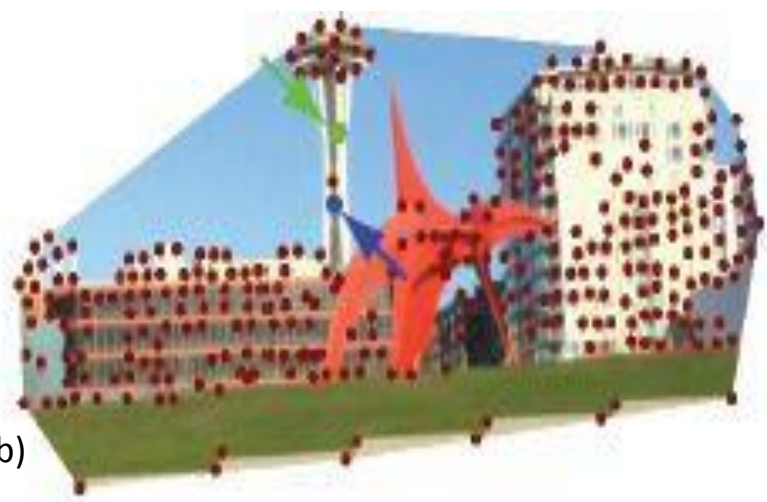

Figure 2.2 Subspace low-pass filtering. Top-left plot: A low-pass filter on two input trajectories (dashed lines) creates very different outputs (solid lines) for two similar trajectories, since their durations are different, leading to broken geometric relationships in the rendered output (a). Top-right plot: if, instead, the trajectory matrix is completed using matrix factorization, the filter outputs are more similar, leading to a better rendered result (b). Images adopted from [5].

If we simply filtered the trajectory matrix directly, it is similar to applying a low pass filter to each trajectory individually. While such an approach does not explicitly constrain the relationships between points, the fact that the filter is linear and applied in the same way to all points implicitly preserves properties of the relationships between points. However, because the matrix $M$ is not complete, the filtering operation is not linear - each point receives different 
treatment (based on its incompleteness), and therefore inter-point relationships are broken. With the subspace constraint, the relationship between features is appropriately preserved [12].

\subsubsection{Bundled Camera Paths Concept}

Liu et al. [7] use bundles of local camera paths to handle non-rigid effects such as rolling shutter while also minimizing geometric distortions. Liu et al. [7] is built on a warping based motion representation, and an adaptive space-time path smoothing. The first component represents the motion between two consecutive frames by mesh-based, spatially-variant homographies as shown in Figure 2.3, with as-similar-as possible regularization constraint $[17,18]$. The second component smooths all bundled camera paths as a whole to maintain both spatial and temporal coherences. As this 2D method approximate cinematography favoured path by adopting a discontinuity-preserving idea similar to bilateral filtering [19], to adaptively control the strength of smoothing, it outperforms other competitive 2D or 3D methods.
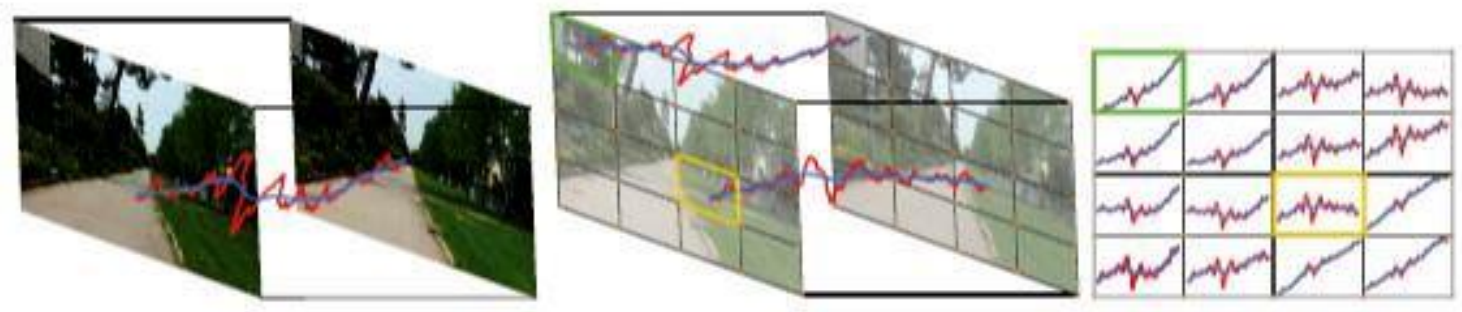

Figure 2.3 Comparison between traditional 2D stabilization (a single global camera path) and bundled camera paths stabilization model.

In this technique, given an original path $\mathrm{C}=\{C(t)\}$, an optimized path $\mathrm{P}=\{P(t)\}$ is obtained by minimizing the following function:

$$
\mathrm{O}(\{P(t)\})=\sum_{t}\left(\|P(t)-C(t)\|^{2}+\lambda_{t} \sum_{r \in \Omega_{t}} \omega_{t, r}(C) .\|P(t)-P(r)\|^{2}\right),
$$

where $\Omega$ t are the neighbourhood at frame t. The other terms are:

- Data term $\|P(t)-C(t)\|^{2}$ enforcing the new camera path to be close to the original one to reduce cropping and distortion;

- Smoothness term $\|P(t)-C(t)\|^{2}$ stabilizing the path; 
- Weight $\omega_{t, r}(C)$ to preserve motion discontinuities under fast panning/rotation or scene transition;

- Parameter $\lambda_{t}$ to balance the above two terms.

In this technique Liu et al. [7] have used a Jacobi-based iterative solver [20] to solve the quadratic equation (2.7).

A bundle of camera paths are generated and are space-time optimized by minimizing the following objective function

$$
\sum_{i} O\left(\left\{P_{i}(t)\right\}\right)+\sum_{t} \sum_{j \in N(i)}\left\|P_{i}(t)-P_{j}(t)\right\|^{2}
$$

where $N(i)$ includes eight neighbors of the grid cell $i$.

After path optimization, the warping matrix $B_{i}(t)$ for each cell $i$ is computed by

$$
B_{i}(t)=C_{i}^{-1}(t) P_{i}(t)
$$

Then $B_{i}(t)$ is applied to warp the $i$-th cell at the $t$-th frame to generate the final output video.

\subsection{3-D Video Stabilization}

3-D video stabilization techniques can achieve high-quality appearance through exploring 3D camera motions and scene depth. From the obtained 3-D shaky camera path, a smoothed virtual path is recovered. The video frames are rendered along the virtual path as if the video was taken from the new, desired camera trajectory. The 3D reconstruction is achieved through structurefrom-motion algorithms (SFM) [25, 26] or by using depth sensors [27]. Some videos do not contain sufficient motion information to allow for reconstruction, in that cases SFM has issues with robustness and generality. But, when 3-D reconstruction is feasible, it often produces the high quality of results due to its physical correctness.

Liu et al. 2009 [2] proposed a 3-D stabilization technique by introducing contentpreserving warps. This techniques was the first that can perform 3-D video stabilization for dynamic scenes. In the following, content preserving warps techniques is briefly reviewed. 


\section{Content-Preserving Warps}

In this method, dynamic content and other temporal properties of video are preserved because each output frame is rendered as a warp of a single input frame. The content-preserving warp was proposed for the novel view synthesis. This method is inspired by as-rigid-as-possible shape manipulation [28], as they are able to minimize perceivable distortion much more effectively than traditional space-warp methods [29].

In this method, given the input video frame $\hat{I}_{t}$, the corresponding output video frame $I_{t}$ is generated by a warp from $\hat{I}_{t}$. 3-D reconstruction provides a sparse set of 3-D points. These points can be projected onto both the input and output cameras, yielding two sets of corresponding 2-D points: $\hat{P}$ on the input and $P$ on the output frame.

A least-squares sense can be computed by discretizing the warp into a grid and minimizing an energy function of two weighted energy terms: a data term for each sparse displacement and a similarity transformation term that measures the deviation of each grid cell from a similarity weighted by the salience of the grid cell.

- Data Term: Suppose $\{p, \hat{p}\}$ is the $p-t h$ matched feature pair from input and output frame respectively. The feature $p$ can be represented by a 2D bilinear interpolation of the four vertices $V_{p}=\left[v_{p}^{1}, v_{p}^{2}, v_{p}^{3}, v_{p}^{4}\right]$ of the enclosing grid cell: $p=V_{p} w_{p}$, where $w_{p}=$ $\left[w_{p}^{1}, w_{p}^{2}, w_{p}^{3}, w_{p}^{4}\right]$ are interpolation weights that sum to 1 . The corresponding feature $\hat{p}$ can be represented by the same weights of the warped grid vertices $\hat{V}_{p}=\left[\hat{v}_{p}^{1}, \hat{v}_{p}^{2}, \hat{v}_{p}^{3}, \hat{v}_{p}^{4}\right]$. Therefore the data term is defined as

$$
E_{d}(\widehat{V})=\sum_{p}\left\|\widehat{V}_{p} w_{p}-\hat{p}\right\|^{2}
$$

Here $\widehat{V}$ contains all the warped grid vertices.

- Similarity transformation term: The similarity transformation term measures the deviation of each output grid cell from a similarity transformation of its corresponding input grid cell. The similarity term is defined as:

$$
E_{S}(\hat{V})=\sum_{\hat{v}}\left\|\hat{v}-\hat{v}_{1}-s R_{90}\left(\hat{v}_{0}-\hat{v}_{1}\right)\right\|^{2}, R_{90}=\left[\begin{array}{cc}
0 & 1 \\
-1 & 0
\end{array}\right],
$$


where $s=\left\|v-v_{1}\right\| /\left\|v_{0}-v_{1}\right\|$ is a known scalar computed from the initial mesh. This similarity transformation term requires the triangle of neighboring vertices $v, v_{0}, v_{1}$ undergoes a similarity transformation.

The final energy $E(\widehat{V})$ is obtained by combining two terms.

$$
E(\widehat{V})=E_{d}(\widehat{V})+\alpha E_{s}(\widehat{V})
$$

where $\alpha$ is a weight to control the amount of regularization. This energy equation is quadratic and can be minimized by solving a sparse linear system.

Content preserving warp is applied to warp a frame to its novel view point. This method is able to achieve more stable and more directed camera motions than the $2 \mathrm{D}$ techniques while avoiding the ghosting of moving scene objects. 


\section{Proposed Video Stabilization}

In this thesis, 2-D motion estimation based video stabilization is implemented. Grundmann et al. [16], Liu et al. 2011 [5] and Liu et al. 2013 [7] relay on estimating features and feature trajectories. So these methods may not handle videos with low feature count, excessive blur during fast motions, or lack of rigid objects in the scene. This thesis attempts to robustly handle most of the consumer level videos and also videos with low feature count and excessive blur in better computation time. The developed video stabilization algorithm pipeline is as shown in Figure 3.1.

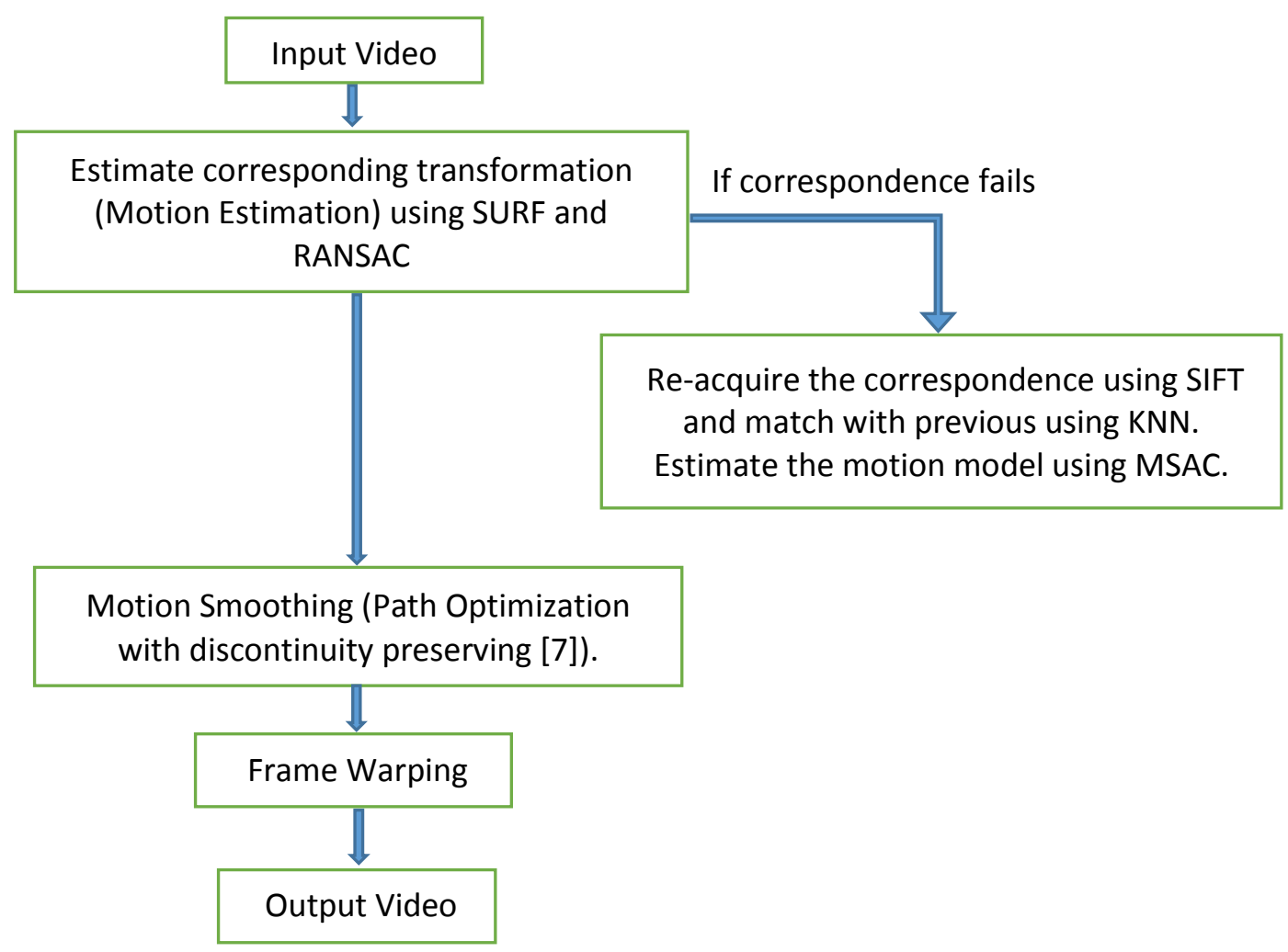

Figure 3.1 Proposed Video Stabilization Pipeline.

We use KNN based feature replacement to maintain the feature count for frames that suffer excessive blur and frames that lack rigid objects in the scene. Also, the motion model selection for such frames is handled using a method of M-estimator sample consensus (MSAC) [30]. In the following, most important feature descriptors that are widely used in computer vision and also 
used in this work are briefly discussed. Then the proposed stabilization method is discussed in detail.

\subsection{Scale Invariant Features (SIFT) and Speeded Up Robust Features (SURF)}

SIFT features are local feature vectors in an image, each of which is invariant to image translation, scaling, and rotation, and partially invariant to illumination changes and affine or 3D projection. The SIFT features share a number of properties in common with the responses of neurons in inferior temporal (IT) cortex in primate vision [31, 32]. Originally these features were proposed for object recognition: features detected in a sample image are matched to a large database of previously extracted features from various objects at different viewpoints. Advantages of invariant local features include,

- Locality: As these features are local, they are robust to occlusion and clutter.

- Distinctiveness: Individual features can be matched to a large database of objects.

- Quantity: Many features can be generated for even small objects (therefore homography estimation is easier).

- Efficiency: These features share a number of properties in common with the responses of neurons in inferior temporal (IT) cortex in primate vision. So they are close to real-time performance.

- Extensibility: Can easily be extended to wide range of differing feature types, with each adding robustness.

The greatest characteristic of SIFT algorithm is scale invariance. To achieve scale invariance, SIFT uses a DoG (Difference of Gaussian) function, formula (3.1), and convolves it on an image. It obtains different scale images by changing $\sigma$. Then, it subtracts the images which are adjacent in the same resolution to get a DoG pyramid.

$$
G(x, y, \sigma)=\frac{1}{2 \pi \sigma^{2}} \exp \left[-\frac{x^{2}+y^{2}}{2 \sigma^{2}}\right]
$$


SIFT compares each point with its adjacent 26 pixels, which is the sum of eight adjacent pixels in the same layer and nine pixels in the upper and lower adjacent layers. If the point is minimum or maximum, the location and scale of this point are recorded. Therefore, SIFT gets all extreme points of DoG scale-space, and locates extreme points exactly. After that, it removes low contrast and unstable edge points. It further removes interference points, using $2 \times 2$ Hessian matrix obtained from adjacent difference images. Next, in the scale of each keypoint, SIFT computes the gradient strength and direction of every neighborhood. According to gradient directions, SIFT votes in histogram for every neighborhood, and uses the summations as the gradient strengths of a keypoint. And the main direction of this keypoint is defined as the direction whose gradient strength is maximal. Then, SIFT uses the keypoint as a center to choose an adjacent 16x16 region. After the region is chosen, SIFT divides this region into $4 \times 4$ sub-regions, and sums the gradient strength in each sub-region. SIFT uses eight directions in each sub-region to generate an eightdimensional vector. Thereby, SIFT gets a 128-dimensional feature description from 16 sub-regions [34].

The basic idea of SURF is similar to that of SIFT, but SURF uses different methods for location detection and descriptor generation. SURF [33] is proposed to improve the detection and description efficiency of extreme points. In SURF, a quick Hessian matrix is adopted for detection, which has competitive advantages on speed and accuracy. Also, an integral image algorithm is adopted to replace the procedure of constructing the Gaussian pyramid in SIFT. Besides, in the description phase, SURF first divides the neighborhood region of each extreme point into a number of $4 \times 4$ square sub-regions. Then, it computes a Haar wavelet response of each sub-region. Each response has a four-dimensional vector. Each keypoint is described with a 64-dimensional feature description of all sub-regions.

SURF features are faster for computation and thus used in this work, SURF are the primary features detected and extracted. But, as SIFT detects the most matches and stable to rotation, video frames with low feature count, excessive blur and lack of rigid objects in the scene are handled using SIFT. 


\subsection{Motion Estimation}

Motion estimation stage estimates the camera path between frames. This work primarily estimates SURF features. SURF features are extracted from two consecutive frames and then these two set of features are matched to obtain a Local Motion Vector for each keypoint. Not all local motion vectors give correct information about how the frame has moved relatively to the previous, so it is necessary to discard wrong matches that do not fit into the estimate transformation and may mislead the process.

\subsubsection{Feature Matching}

Feature matching is an important problem to address for motion estimation. As proposed in [31], Euclidean distance between feature descriptor vectors and a distance ratio, namely ratio of closest neighbour distance to that of the second-closest one, that can be checked against a threshold to discard false matches. In fact correct matches should have lower ratios while wrong ones should have ratios closer to one.

The minimum number of matches required to solve a homogenous linear equation system (for the further model fitting) is four. It has been observed that for frames with excessive blur and that lack rigid objects in the scene, the number of detected features are below four. This is because SURF doesn't work well in excessive rotation; it finds the least matches and gets the least repeatability. And feature matching and further processing is not attained. So in this work, such frames are addressed by detecting SIFT features; as SIFT detects the most matches and stable to rotation. SIFT is only used for specific frames because we want our method to be computationally optimal. SURF can improve the performance as it is speeded and robust for extreme points. Also all the video frames don't have excessive blur or lack rigid objects. After extracting SIFT features, based on the local motion vectors of the previous frames, N-nearest neighbour are matched to the detected features. If the frames are too blurry for SIFT descriptors, then based on the feature point location in the previous frame a local window of features in the current frame are obtained using local motion vector and are matched using N-nearest neighbors [36]. Minkowski distance metric is used while searching for the N-nearest neighbors, for more details refer [37, 38, 39]. 


\subsubsection{2-D Homography Estimation}

A common motion estimation approach for video stabilization is to estimate a dominant planar homography that stabilizes a large planar region in the video.

\section{Direct Linear Transformation Algorithm}

The Direct Linear Transform (DLT) algorithm is an algorithm used to solve for the homography matrix $H$ given a sufficient set of point correspondences. The relationship between two corresponding points $\boldsymbol{x}$ and $\boldsymbol{x}^{\prime}$ can be written as [40]:

$$
\begin{gathered}
c\left(\begin{array}{l}
u \\
v \\
1
\end{array}\right)=H\left(\begin{array}{l}
x \\
y \\
1
\end{array}\right), \\
x^{\prime}=H x
\end{gathered}
$$

where c is any non-zero constant, $\boldsymbol{x}^{\prime}$ is represented as $\left(\begin{array}{lll}u & v & 1\end{array}\right)^{T}, \boldsymbol{x}$ is represented as $\left(\begin{array}{lll}x & y & 1\end{array}\right)^{T}$, and $H=\left(\begin{array}{lll}h_{1} & h_{2} & h_{3} \\ h_{4} & h_{5} & h_{6} \\ h_{7} & h_{8} & h_{9}\end{array}\right)$.

Dividing the first row of equation (3.2) by the third row and the second row by the third row we get the following two equations:

$$
\begin{aligned}
& -h_{1} x-h_{2} y-h_{3}+\left(h_{7} x+h_{8} y+h_{9}\right) u=0 \\
& -h_{4} x-h_{5} y-h_{6}+\left(h_{7} x+h_{8} y+h_{9}\right) v=0
\end{aligned}
$$

Equations (3.4) and (3.5) cam be written in matrix form as:

$$
A_{i} \boldsymbol{h}=0
$$




$$
\begin{array}{rlll}
\text { where } & A_{i}= & \left(\begin{array}{cccccccccc}
-x & -y & -1 & 0 & 0 & 0 & u x & u y & u \\
0 & 0 & 0 & -x & -y & -1 & v x & v y & v
\end{array}\right) \quad \text { and } \quad \boldsymbol{h}= \\
\left(\begin{array}{llllllllll}
h_{1} & h_{2} & h_{3} & h_{4} & h_{5} & h_{6} & h_{7} & h_{8} & h_{9}
\end{array}\right)^{T} &
\end{array}
$$

The reason for needing at least 4 corresponding matches, as stated earlier is as explained: since each point correspondence provides 2 equations, 4 correspondences are sufficient to solve for the 8 degrees of freedom of $H$. The restriction is that no 3 points can be collinear (i.e., they must all be in "general position"). Four $2 \times 9 A_{i}$ matrices (one per point correspondence) can be stacked on top of one another to get a single $8 \times 9$ matrix $A$. The $1 \mathrm{D}$ null space of $A$ is the solution space for $\boldsymbol{h}$.

In many cases we may be able to use more than 4 correspondences to ensure a more robust solution. However many point correspondences are used, if all of them are exact then $A$ will still have rank 8 and there will be a single homogeneous solution. In practice, there will be some uncertainty, the points will be inexact and there will not be an exact solution. The problem then becomes to solve for a vector $h$ that minimizes a suitable cost function. Minimize ||$A \boldsymbol{h} \|$ s. $t .|| \boldsymbol{h} \|=$ 1 , which is equivalent to minimize $\boldsymbol{h}^{T} A^{T} A \boldsymbol{h}$ s.t. || $\boldsymbol{h}||=1$. The solution is eigenvector $A^{T} A$ associated with the smallest eigenvalue. DLT algorithm can lead to unstable results because of the numerical calculation, inaccurate point correspondences and so on. This motivates a robust algorithm, such as RANSAC [41].

\section{Normalization}

For the numerical calculation issues in the DLT algorithm, a normalization process should be applied. This step is very important for less well conditioned problems such as DLT. [4] assert that the result of the DLT algorithm as presented above is dependent on the origin and scale of the coordinate system in the image. This is a very undesirable property as it makes the algorithm quite unstable. The reason for this non-invariance has to do with how the DLT method uses the SVD of $A$ to obtain a solution to the overdetermined set of equations $A \boldsymbol{h}=0$, for more details refer [42]. But the main message is that for exact data and infinite precision the result is fine but in the presence of noise the solution typically diverges from the correct result. 
To ensure that the solution converges to the correct result even in the presence of noise, Hartley and Zisserman [4] propose a normalization step, which is used in this thesis. Their normalized DLT algorithm works as follows:

1. Compute a similarity transform $T$ that takes points $\boldsymbol{x}_{\boldsymbol{i}}$ to a new set of points $\tilde{\boldsymbol{x}}_{\boldsymbol{i}}$ such that the centroid of the points $\widetilde{\boldsymbol{x}}_{i}$ is the coordinate origin and their average distance from the origin is $\sqrt{2}$.

2. Compute a similar transformation $T^{\prime}$ transforming points $\boldsymbol{x}_{\boldsymbol{i}}{ }^{\prime}$ to $\widetilde{\boldsymbol{x}}_{i}{ }^{\prime}$.

3. Apply the DLT algorithm from above using $\boldsymbol{x}_{\boldsymbol{i}}{ }^{\prime}$ and $\widetilde{\boldsymbol{x}}_{\boldsymbol{i}}{ }^{\prime}$ to obtain homography matrix $\widetilde{H}$.

4. Compute $H=\left(T^{\prime}\right)^{-1} \widetilde{H} T$.

\subsubsection{Robust Estimation using RANSAC and MSAC}

Many of the point correspondences obtained in the previous step are incorrect. So outlier rejection is important for robust estimation. The two robust methods for outlier rejection RANSAC and MSAC, which are used in this work are briefly discussed.

\section{Random sample consensus (RANSAC)}

Random sample consensus (RANSAC) [41] is an iterative method to estimate parameters of a mathematical model from a set of observed data which contains outliers. It was first published in 1981 to solve the Location Determination Problem (LDP), where the goal is to determine the points in the space that project onto an image into a set of landmarks with known locations. Now it is the most commonly used robust estimation method for homographies [43].

RANSAC is a general framework for model fitting in the presence of outliers. It takes an iterative approach to find the best model for a set of data by generating a hypothesis from random samples and verifying it to the data. The idea of the algorithm is as follow: For a number of iterations, a random sample of 4 correspondences is selected and a homography $H$ is computed from those four correspondences. Each other correspondence is then classified as an inlier or outlier depending on its concurrence with $H$. After all of the iterations are done, the iteration that contained the largest number of inliers is selected. $H$ can then be recomputed from all of the correspondences that were consider as inliers in that iteration. 
Some parameters has to be tuned to decide how to classify correspondence as inlier or outlier. Statistically speaking, the goal is to assign a distance threshold $t$, (between $\boldsymbol{x}^{\prime}$ and $H \boldsymbol{x}$ for example), such that with a probability $\alpha$ the point is an inlier.

Another parameter to decide is the number of iteration to run the algorithm. It will likely be infeasible to try every combination of 4 correspondences, and thus the goal becomes to determine the number of iterations, $N$, that ensures with a probability $p$ that at least one of the random samples will be free from outliers. Hartley and Zisserman [4] gives an equation for $N$ as shown below,

$$
N=\log (1-p) / \log \left(1-(1-\epsilon)^{S}\right)
$$

where $\epsilon$ is the probability that a sample correspondence is an outlier and $s$ is the number of correspondences used in each iteration, which is 4 in this case. If $\epsilon$ is unknown, the data can be probed to adaptively determine $\epsilon$ and $\mathrm{N}$.

In this work, as stated above, number of correspondences used in each iteration $s=4$ and are randomly sampled from the obtained correspondence. Distance threshold is chosen to be $t=$ 0.001 , probability $p$ that at least one of the random samples will be free from outliers $p=0.99 . N$ and $\epsilon$ are determined in every iteration based on the data as stated above.

\section{M-Estimator Sample Consensus (MSAC)}

RANSAC can be sensitive to the choice of the correct noise threshold that defines which data points fit a model instantiated with a certain set of parameters [47]. If such threshold is too large, then all the hypotheses tend to be ranked equally (good). On the other hand, when the noise threshold is too small, the estimated parameters tend to be unstable (i.e. by simply adding or removing a datum to the set of inliers, the estimate of the parameters may fluctuate). To partially compensate for this undesirable effect, MSAC (M-estimator SAmple and Consensus) was proposed. The aim of M-estimators is to alter the influence of outlying values to make the distribution conform to Gaussian assumptions. RANSAC does not use complex optimization as like M-estimator. Usually there is a trade-off between the number of inliers in the model and the scale of the noise. RANSAC has constant loss at large error, while MSAC (M-estimator SAC) 
adopts bounded loss of RANSAC. M-Estimator Sample Consensus formulation is not detailed in this work.

Robust estimation for frames with excessive blur and lack rigid objects in the scene is performed using MSAC; as more inlier correspondence is achieved. For additional information about MSAC, refer [30, 44, 45, 46 and 47].

\subsection{Motion Smoothing}

The estimated motion parameters are forwarded to motion compensation, which damps camera motion by removing high-frequency fluctuations and computes the global transformation necessary to stabilize the current frame. This thesis follow the path optimization idea of Liu et al. 2013 [7]. After motion smoothing final image composition warps the current frame according to that transformation and generates the stabilized sequence.

\subsubsection{Path Optimization}

An optimization based framework that consider multiple competing factors like, removing jitters, avoiding excessive cropping, and minimizing various geometrical distortions (shearing/skewing, wobble), was proposed by Liu et al. 2013 [7].

\section{Formulation}

Given an original path $\mathbf{C}=\{C(t)\}$, an optimized path $\mathbf{P}=\{P(t)\}$, is obtained by minimizing the following function:

$O(\{P(t)\})=\sum_{t}\left(\|P(t)-C(t)\|^{2}+\lambda_{t} \sum_{r \in \Omega_{t}} \omega_{t, r}(\mathbf{C}) .\|P(t)-P(r)\|^{2}\right)$,

where $\Omega$ t are the neighbourhood at frame t. The other terms are:

- Data term $\|P(t)-C(t)\|^{2}$ enforcing the new camera path to be close to the original one to reduce cropping and distortion;

- Smoothness term $\|P(t)-C(t)\|^{2}$ stabilizing the path;

- Weight $\omega_{t, r}(\mathbf{C})$ to preserve motion discontinuities under fast panning/rotation or scene transition; 
- Parameter $\lambda_{t}$ to balance the above two terms.

As equation (3.8) is quadratic, Jacobi-based iterative solver [20] is used to solve it.

$$
P^{(\xi+1)}(t)=\frac{1}{\gamma} C(t)+\sum_{r \in \Omega_{t}, r \neq t} \frac{2 \lambda_{t} \omega_{t, r}}{\gamma} P^{(\xi)}(r)
$$

where $\gamma=1+2 \lambda_{t} \sum_{r \in \Omega_{t}, r \neq t} \omega_{t, r}$, and $\xi$ is an iteration index.

\section{Discontinuity-preserving}

The adaptive weights $\omega_{t, r}$ is tuned to preserve the motion discontinuity by following the idea of bilateral filter [19].

$$
\omega_{t, r}=G_{r}(|| C(r)-C(t) \|)
$$

where $G_{r}$ is the Gaussian function given by:

$$
G_{r}=\frac{1}{\sigma \sqrt{2 \pi}} e^{\frac{-r^{2}}{2 \sigma^{2}}}
$$

\subsubsection{Warping Transformation}

At initialization, $P^{(0)}(t)=C(t)$ and $\Omega_{t}$ is set to 25 . Once the optimized camera path $\mathbf{P}$ is obtained, the warping transformation is computed as

$$
B(t)=C^{-1}(t) P(t)
$$

\section{Cropping and Distortion Control}

The above equation $\omega_{t, r}$ can give a certain amount of ability to control cropping and distortion. However, if the user want to have strict control on the cropping and distortion, a constrained optimization has to be formulated. But, it may be too complex to be solved. Liu et al. 2013 [7] resort to a simple but effective method (adaptively adjust the parameter $\lambda_{t}$ for each frame) for the optimization. This work adopts the idea of adaptively adjusting the parameter $\lambda_{t}$ for each frame. At first the global fixed $\lambda_{t}=\lambda$ (empirically set to 5) to run the optimization and then check the cropping ratio and distortion of every frame. For any frame that does not satisfy the user requirements (cropping ratio or distortion is smaller than a pre-defined threshold), $\lambda_{t}$ is decreased 
by a step $\left(1 / 10 \lambda_{t}\right)$ (according to Equation 3.9, a smaller $\lambda$ will make the optimized path closer to the original one, which has less cropping and distortions) and re-run the optimization. This procedure is repeated until all frames satisfy the requirements.

\subsection{Experimental Results}

The algorithm is initially developed using MATLAB - R2012b on an Intel Core i7 3.4 $\mathrm{GHz}$ machine. Later few of developed functions are converted to Object Oriented Programming (with classes and methods) using MATLAB - R2015b version. For the categorised video datasets with a video resolution of $360 \times 640$, the average video durations, the proposed algorithm average run-times compared to Deshaker software are as shown in the Table 3.1,

\section{Table 3.1 The average run-times for the proposed algorithm for a video duration around}

10 to 30 secs. 19 video sequences of each category are used in the experiment.

\begin{tabular}{|c|c|c|c|}
\hline $\begin{array}{c}\text { Video data } \\
\text { category }\end{array}$ & $\begin{array}{c}\text { Average video } \\
\text { duration }\end{array}$ & $\begin{array}{c}\text { Proposed algorithm } \\
\text { average run-time }\end{array}$ & $\begin{array}{c}\text { Deshaker average } \\
\text { run-time }\end{array}$ \\
\hline Simple & $10 \sim 30$ secs & $37 \sim 141$ secs & $99 \sim 340$ secs \\
\hline Rotation & $10 \sim 30$ secs & $27 \sim 158$ secs & $75 \sim 399$ secs \\
\hline Zooming & $10 \sim 20$ secs & $43 \sim 105$ secs & $150 \sim 324$ secs \\
\hline Parallax & $10 \sim 30$ secs & $53 \sim 84$ secs & $118 \sim 263$ secs \\
\hline Crowd & $10 \sim 30$ secs & $67 \sim 138$ secs & $189 \sim 476$ secs \\
\hline Running & $10 \sim 12$ secs & $50 \sim 69$ secs & $161 \sim 216$ secs \\
\hline
\end{tabular}

As there is no publicly available implementation of video stabilization algorithm of previous works, the results are compared to the well-known commercial systems and free software. Figure 3.1 shows the stabilized ouput frame of a video with quick rotation. (a) is the result with normal 2-D motion estimation and warping. (b) is the result of testing if the frame has excessive rotation, and then adaptively estimating the motion using SIFT and MSAC and warping. (c) is the 
result of Deshaker software. (d) is the result of YouTube video editor. The Figure 3.2 shows that the algorithm is performing as good as well-known stabilization systems.
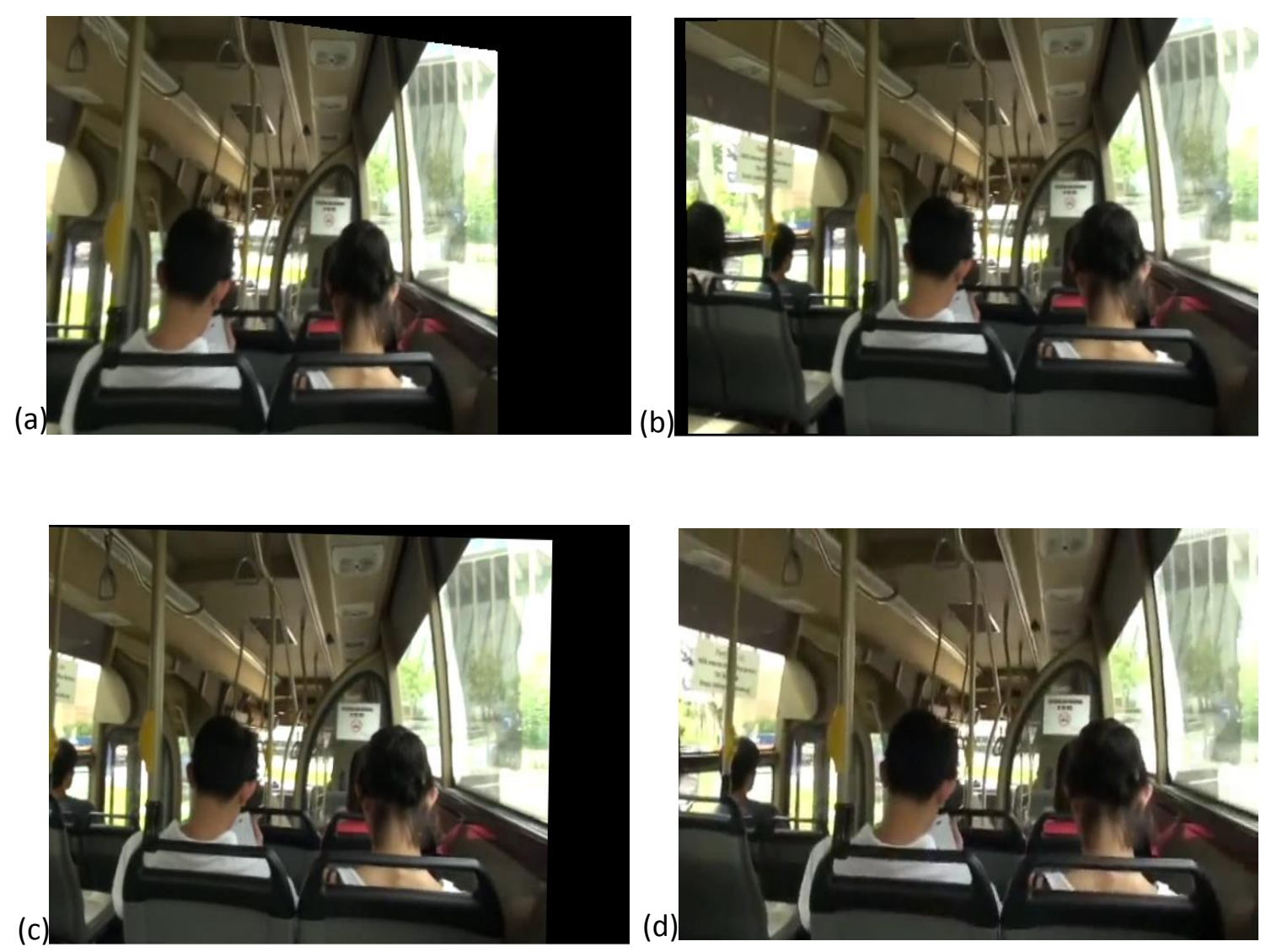

Figure 3.2 Comparative results of the proposed algorithm (b) with the Deshaker software

(c) and the YouTube video editor (d).

For the enlightenment of reader, temporal slices of the unstable video and the stabilized output videos using our algorithm and Deshaker software in a video with crowded scene are presented in Figure 3.3. Image slice to the right in Figure 3.3 shows the result of Deshaker software, which still have some jitters. One of it is pointed out for comparison. Figure 3.4 shows more temporal slices using the proposed video stabilization method. 

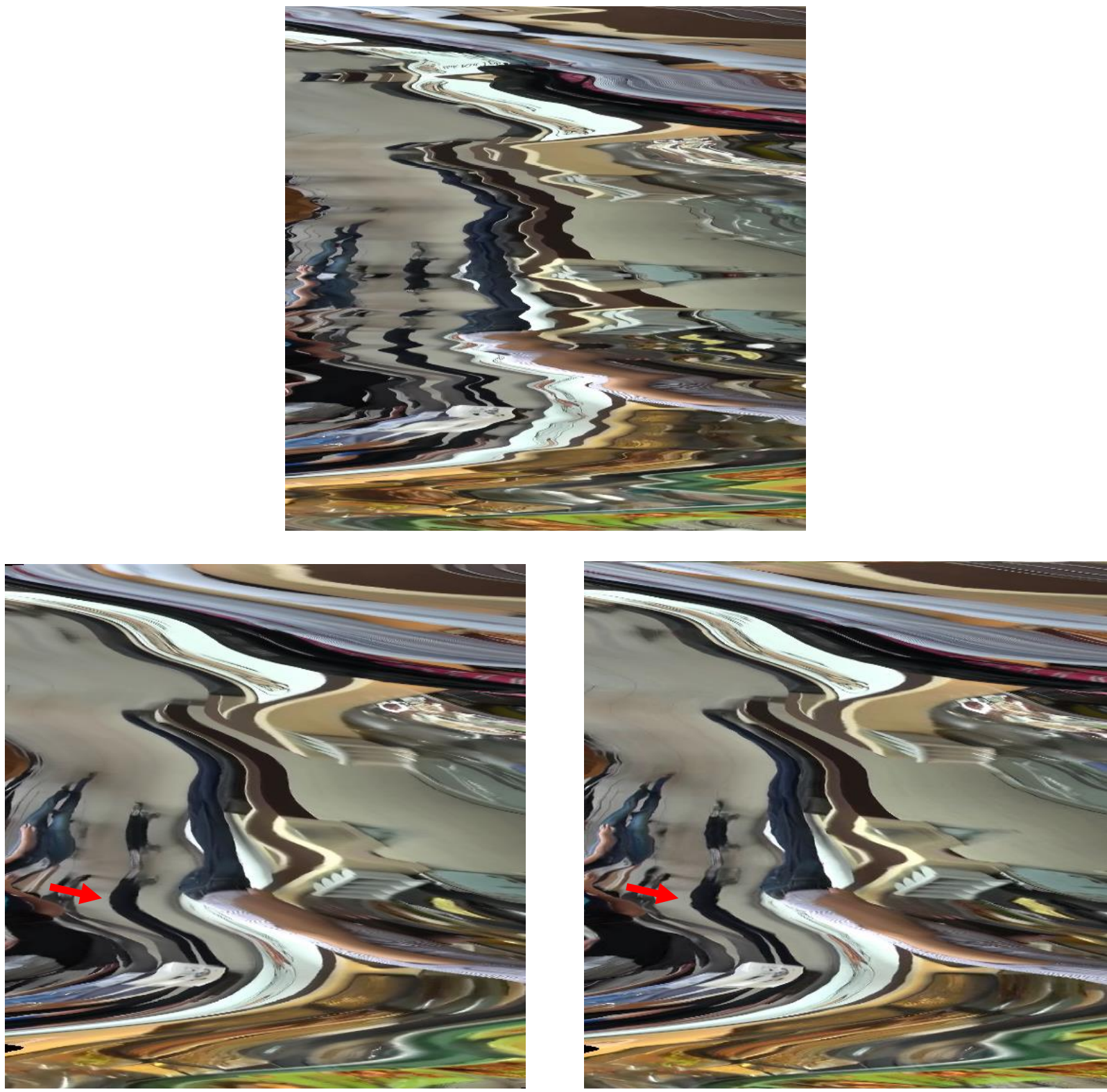

Figure 3.3 Top most figure shows a temporal slice from unstable video. Figure to the left shows corresponding slice in the stabilized video using our algorithm. Figure to the right is the result of Deshaker software. 

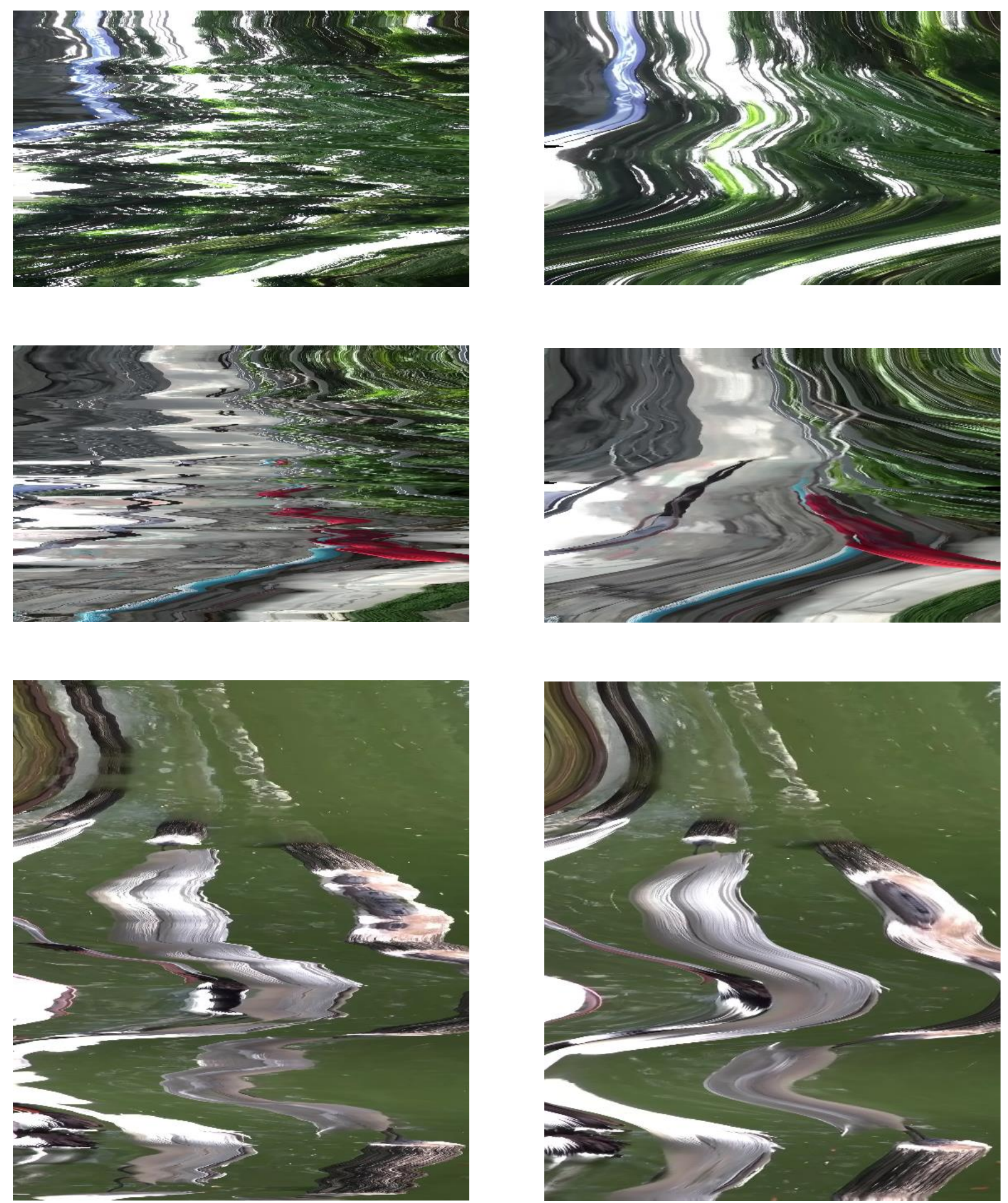

Figure 3.4 Images to the left are temporal slices of video with jittering. Images to the right are slices of stabilized video using our algorithm. 


\section{Proposed Quality Evaluation Metric}

So far in the literature, the video stability (jittering in video) is evaluated by subjective (visual perception) comparison of the original and the stabilized video. Qualitative assessment for the video stability is not studied so far. This work proposes a new quality evaluation metric, which estimates the jitteriness in the video and thus helps in visualizing the camera motion in unstable and stable video data. The proposed quality evaluation metric is a total-variation based score estimated by tracking the salient image features such as corners. The corner features are selected for tracking because the correspondence of such features from frame to frame is high. While other features are easily subject to intensity variations, occlusions, etc., and other factors that result in incorrect correspondence by the tracking algorithm. The developed quality evaluation algorithm pipeline is as shown in Figure 4.1.

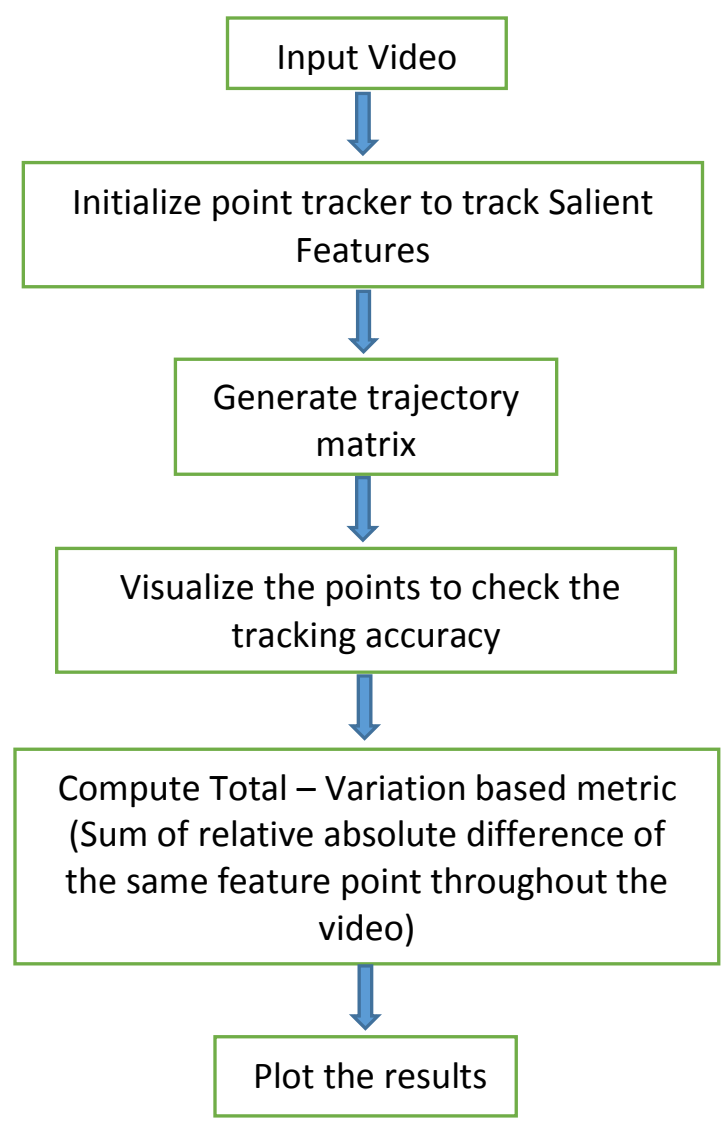

Figure 4.1 Proposed Quality Evaluation Pipeline. 
The approach we followed to calculate the jittering is straightforward, which is to estimate the camera motion though the capture of video. The camera motion is computed by generating the trajectory matrix, by tracking the salient features through the video frames using KLT (KanadeLucas-Tomasi) feature tracker. During the progress of tracking, if a feature point disappears, it is replaced by reacquiring features and assigning the lost feature with a nearest reacquired feature using KNN search. In the following, the proposed quality metric is discussed in details.

\subsection{Corner Detector}

Harris and Stephens (1998) [49] proposed a combined corner and edge detector based on the local auto-correlation function. They have defined a corner to be a point with low selfsimilarity. The algorithm tests each pixel in the image to see if a corner is present. Corners are considered to be the regions in image with large variation in intensity in all the directions. The feature detector is usually called corner detector and is not selecting just corners, but rather any image location that has large gradients in all directions at a predetermined scale. [49] Harris Corner Detector finds the difference in image intensity for a displacement of $(u, v)$ in all directions. A window function (either a Rectangle or Gaussian window) is multiplied over the difference of image intensity. In the given window patch, if there is a significant intensity change in all directions, then a corner is detected Figure 4.2.
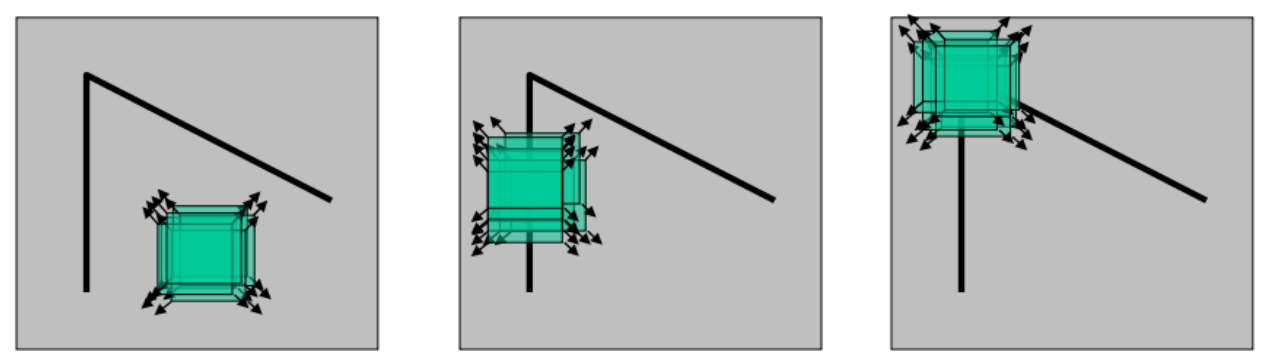

\section{Figure 4.2 Corner pixel has surroundings different from all its neighbors in all directions.}

The corner detector algorithm depend on the eigenvalues of the summation of the squared difference matrix (SSD). The eigenvalues of an SSD matrix represent the differences between the surroundings of a pixel and the surroundings of its neighbors. The larger the difference between the surroundings of a pixel and those of its neighbors, the larger the eigenvalues. The larger the eigenvalues, the more likely that a pixel appears at a corner. 
Rosen \& Drummond (2006) [48] proposed a high-speed method for corner detection using local intensity comparison, called Features from Accelerated Segment Test (FAST). This method is said to be faster and computationally efficient compared to Harris and SIFT (Difference of Gaussians) feature extraction methods discussed earlier. FAST corner detector uses a circle of 16 pixels around a corner candidate $p$ to classify whether it is actually a corner. If a set of $n$ contiguous pixels in the circle are all brighter than the intensity of candidate pixel $p$ (denoted by $I p$ ) plus a threshold value $t$, or all darker than the intensity of candidate pixel $p$ minus threshold value $t$ (Ip $t$ ), as shown in Figure 4.3, then $p$ is classified as corner.

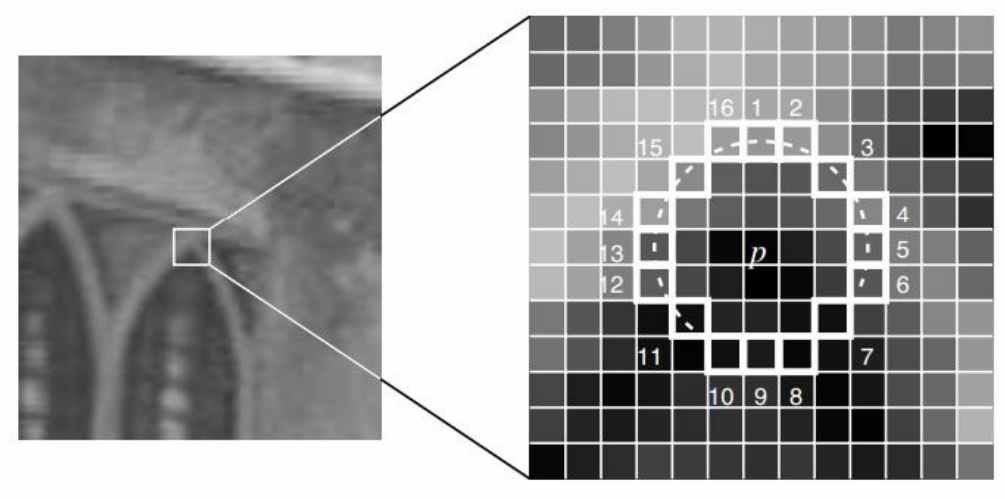

Figure 4.3 12 point segment test corner detection in an image patch. The highlighted squares are the pixels used in the corner detection. The pixel at $\boldsymbol{p}$ is the centre of a candidate corner. The arc is indicated by the dashed line passes through 12 contiguous pixels which are brighter than $p$ by more than the threshold. Image adopted from [48].

The high-speed test for rejecting non-corner points is operated by examining 4 example pixels, namely pixel 1,9,5 and 13. Because there should be at least 12 contiguous pixels that are whether all brighter or darker than the candidate corner, so there should be at least 3 pixels out of these 4 example pixels that are all brighter or darker than the candidate corner. Firstly pixels 1 and 9 are examined, if both $I 1$ and $I 9$ are within $[I p-t, I p+t]$, then candidate $p$ is not a corner. Otherwise pixels 5 and 13 are further examined to check whether three of them are brighter than $I p+t$ or darker than Ip $-t$. If there exists 3 of them that are either brighter or darker, the rest pixels are then examined for final conclusion. 
However, there are several weaknesses for this test method:

1. The high-speed test cannot be generalized well for $n<12$. If $n<12$, it would be possible that a candidate $p$ is a corner and only 2 out of 4 example test pixels are both brighter Ip + $t$ or darker than $I p-t$.

2. The efficiency of the detector depends on the choice and ordering of these selected test pixels. However it is unlikely that the chosen pixels are optimal which take concerns about the distribution of corner appearances.

3. Multiple features are detected adjacent to one another.

In this work, [48] is used for corner detection as it is faster compared to [49] and experimentally [48] detected more corners than [49]. The threshold is set to $10^{-5}$. The corner and edge features are detected from the first video frame and tracked through the other frames of the video using KLT tracker, which is briefed in the following.

\subsection{Point Tracking}

Tracking deals with estimating the trajectory of a feature or object in the image plane as it moves through the frames. The point tracking algorithms track set of $N$ features initialized for tracking. This work uses the standard KLT (Kanade-Lucas-Tomasi) tracker with $N$ initialized $(N=300$ in this case) FAST [48] corner feature points.

The KLT tracker is based on the early work of Lucas and Kanade [51], was developed fully by Tomasi and Kanade [52], and was explained clearly in the paper by Shi and Tomasi [50]. Briefly, good features are located by examining the minimum eigenvalue of each 2 by 2 gradient matrix, and features are tracked using a Newton-Raphson method of minimizing the difference between the two windows. Multiresolution tracking allows for relatively large displacements between images. The simple tracking algorithm implemented in this work is described in the following steps,

1. Find corner features by local intensity comparison (Features from Accelerated Segment Test, FAST [48]) method. 
2. For each corner feature, compute motion (translation or affine) between consecutive frames.

3. Link motion vectors in successive frames to get a track for each feature.

4. Check if the features is lost in the successive tracking, if it is lost, introduce a new FAST feature by applying the corner detector and replacing the old feature with nearest new feature using Nearest Neighbor search.

5. Track new and old feature points using steps 1-3.

6. Generate trajectory matrix with all the obtained feature tracks.

\subsection{Point Track Visualization}

Generated feature tracks are visualized to check the tracking accuracy. A matrix of different color shades (255) is generated using MATLAB and each feature trajectory is plotted through the video frames as shown below in Figure 4.4. In Figure 4.4 (a), it can be noticed that as the bus moves, the feature tracks at the bus tires and the window also shifted. In the corresponding frames, when the bus move out of the frame, those points are lost and new points are acquired and replaced. In Figure 4.4 (b), the frame to the right is a quick zoom - in of the frame to the left, it can be noticed that old feature points are replaced to new nearest neighboring corner points. 
(a)
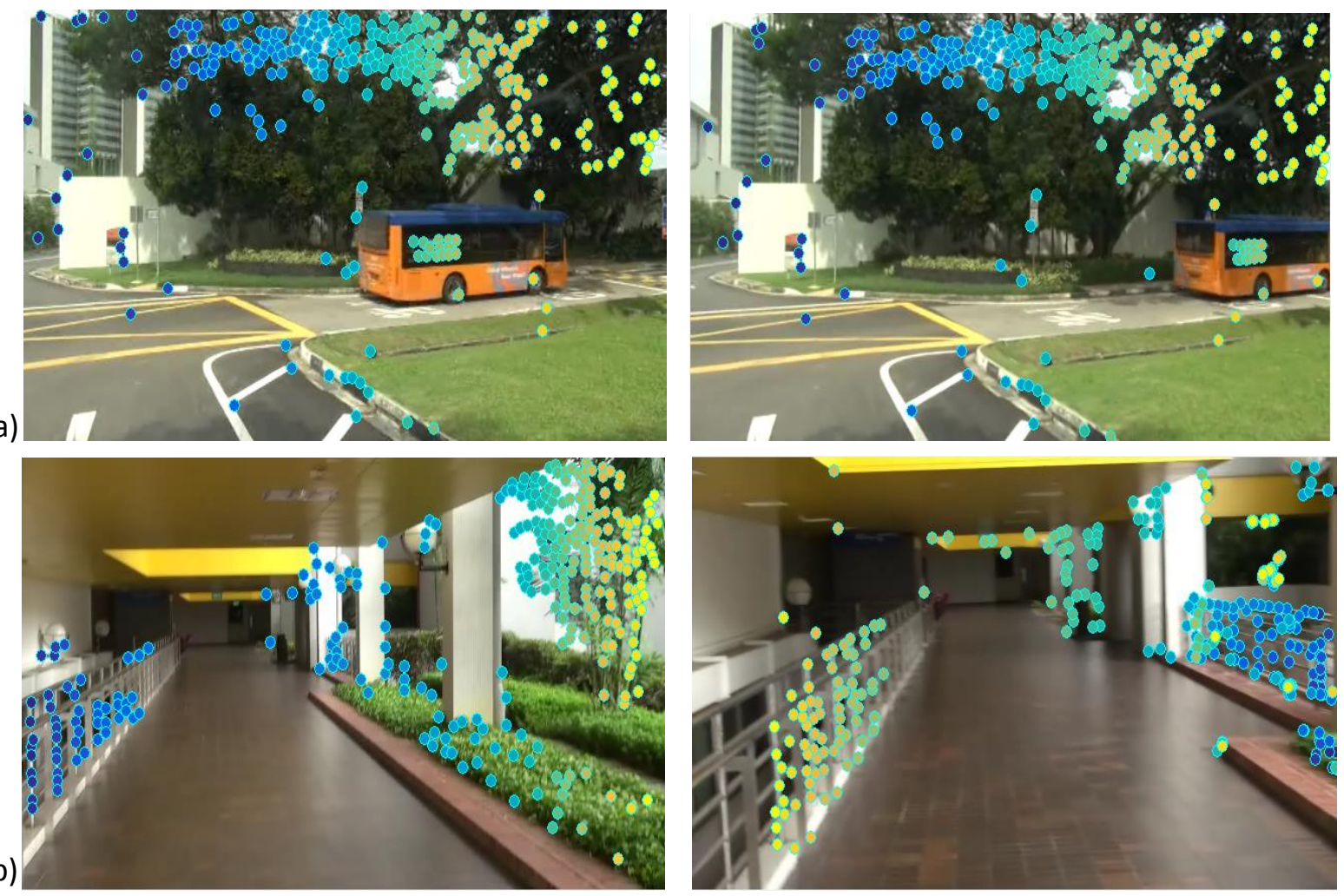

Figure 4.4 Visualization of the feature tracks.

\subsection{Metric Evaluation}

A total-variation based metric is proposed and developed in this work. In mathematics, roughly speaking, total variation measure is an infinitesimal version of the absolute value. The total variation has been introduced in Computer Vision first by Rudin, Osher and Fatemi [53], as a regularizing criterion for solving inverse problems. It has proved to be quite efficient for regularizing images without smoothing the boundaries of the objects. In classical analysis, the total variation of a function $f$ over an interval $[a, b]$ is defined as in equation 4.1.

$$
v(f,[a, b]):=\sup _{\mathrm{g}} \sum_{i=1}^{k}\left|f(t)-f\left(t_{i-1}\right)\right|
$$

where the supremum runs over all finite grids $\mathfrak{g}: a=t_{0}<t_{1}<\cdots t_{k}=b$ on $[a, b]$. 
The focus of this work is to find the absolute value for the $i-t h$ feature trajectory, where $\left(x_{t}^{i}, y_{t}^{i}\right)$ is the $i-t h$ feature point in frame $t$. If we have a set of $N$ feature trajectories across $F$ frames, then the trajectory matrix $T$ is given by,

$$
T_{N \times 2 F}=\left[\begin{array}{cccccc}
x_{1}^{1} & y_{1}^{1} & & x_{F}^{1} & y_{F}^{1} \\
x_{1}^{2} & y_{1}^{2} & & x_{F}^{2} & y_{F}^{2} \\
& \vdots & & \ddots & & \vdots \\
x_{1}^{N-1} & y_{1}^{N-1} & & x_{F}^{N-1} & y_{F}^{N-1} \\
x_{1}^{N} & y_{1}^{N} & & x_{F}^{N} & & y_{F}^{N}
\end{array}\right]
$$

Now the relative absolute difference for the same feature point throughout the video is computed as follow,

$$
T . V=\sum_{i \rightarrow 1-N, t \rightarrow 1-F}\left|\left(x_{t}^{i}-x_{t+1}^{i}\right)+\left(y_{t}^{i}-y_{t+1}^{i}\right)\right|
$$

Relative scores of all the feature points in a frame are averaged to compute the absolute path. In this way, the jittering in the camera path is estimated and the total score is plotted. In the following, the experimental results with the applied quality evaluation matric on the unstable and stabilized videos is discussed.

\subsection{Experimental Results}

The proposed quality evaluation metric is tested on the unstable videos from the categorised dataset and the stabilized videos using the proposed video stabilization method, Deshaker and YouTube [6]. The experimental results demonstrate two things. First, the proposed quality evaluation metric nicely estimates the camera path and helps visualize and compare the jittering before and after stabilization. Second, the proposed robust video stabilization algorithm is not only performing subjectively good, but also qualitatively compared to the available commercial software. The plots below objectively visualize the jittering in the camera motion. Figure 4.5 and 4.6 shows the camera jittering in normal videos (that do not require any stabilization), captured using Samsung Note 5. Figure 4.7 to 4.11 shows the camera jittering in one of the videos from each categorized shaky video dataset. 


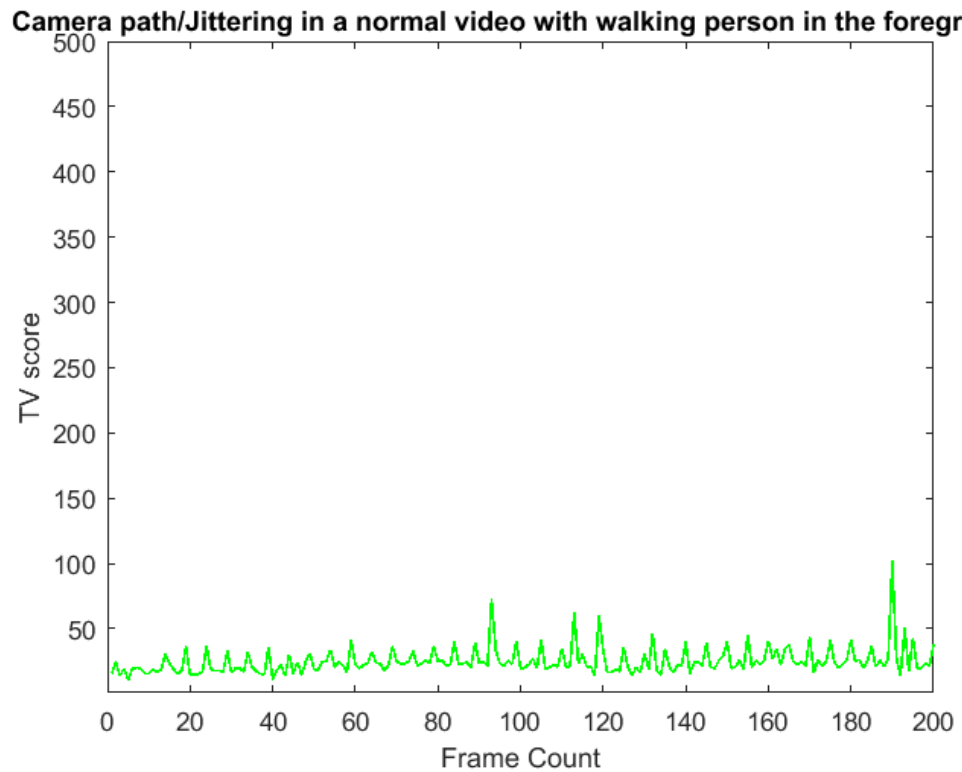

Figure 4.5 TV plot in a normal video (stable video) with walking person in the foreground.

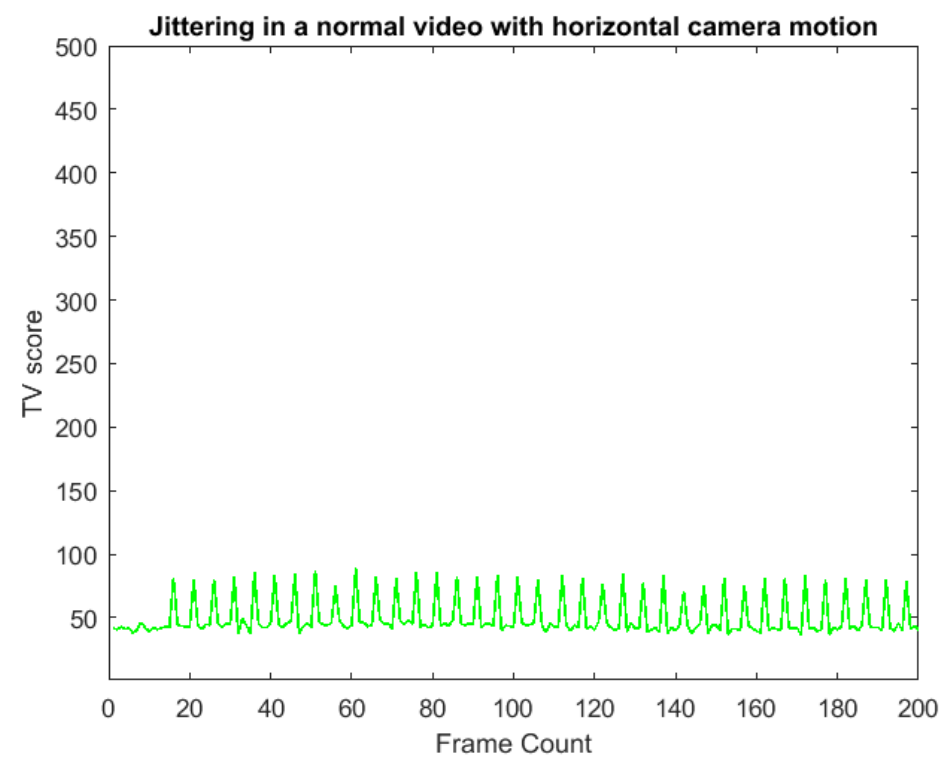

Figure 4.6 TV plot in a normal video with constant horizontal camera motion. 


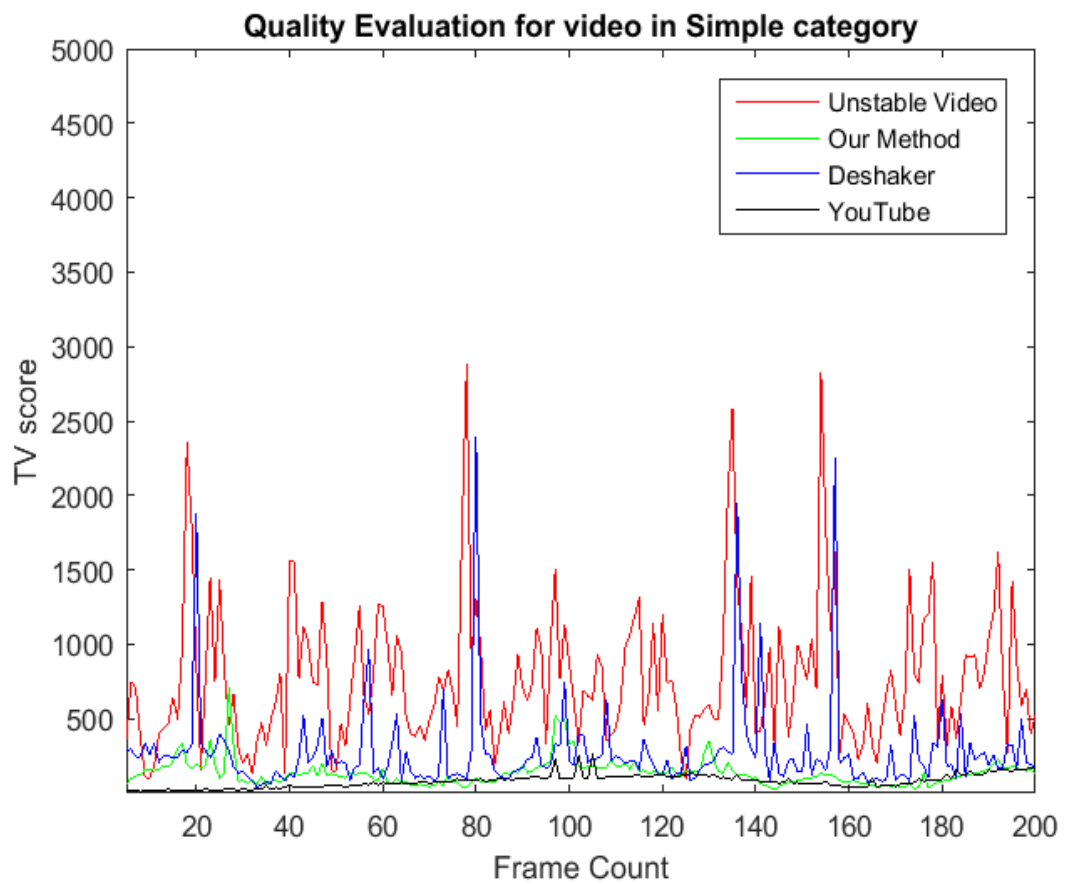

Figure 4.7 Jittering in the camera motion for unstable video in Simple category, compared to the stabilised videos.

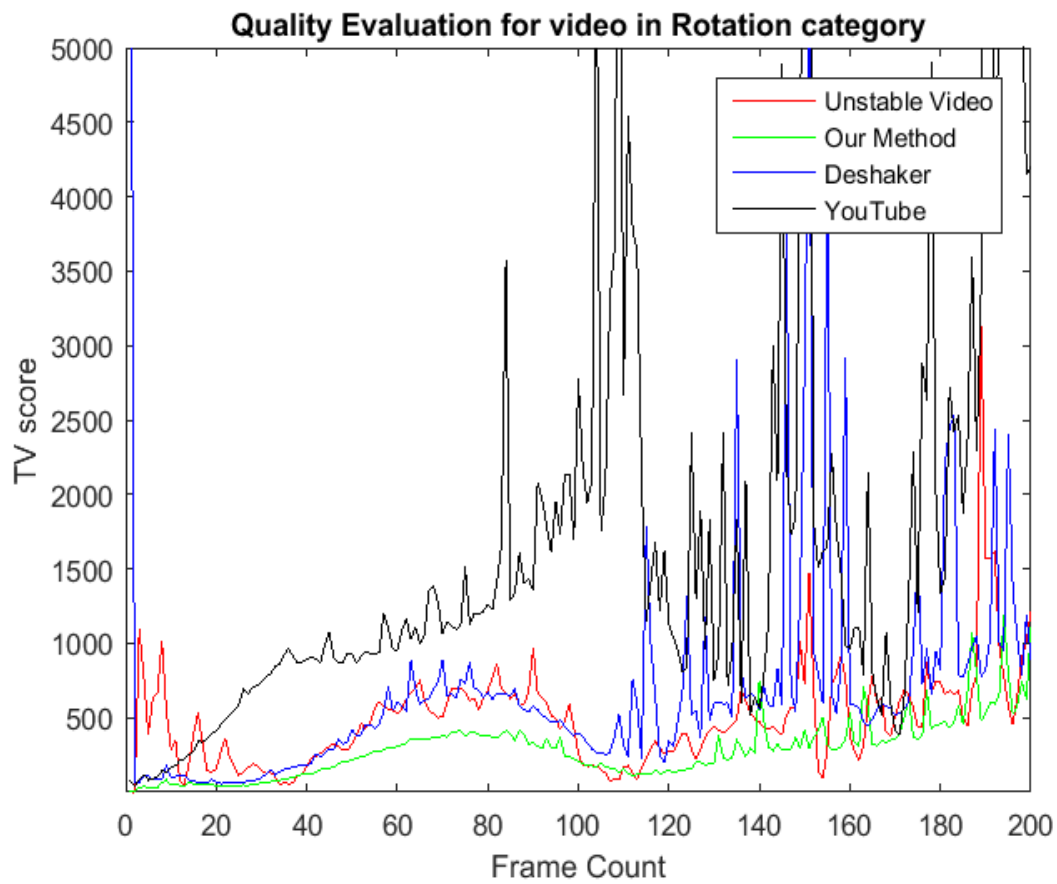

Figure 4.8 Jittering in the camera motion for unstable video with quick rotation, compared to the stabilised videos. 


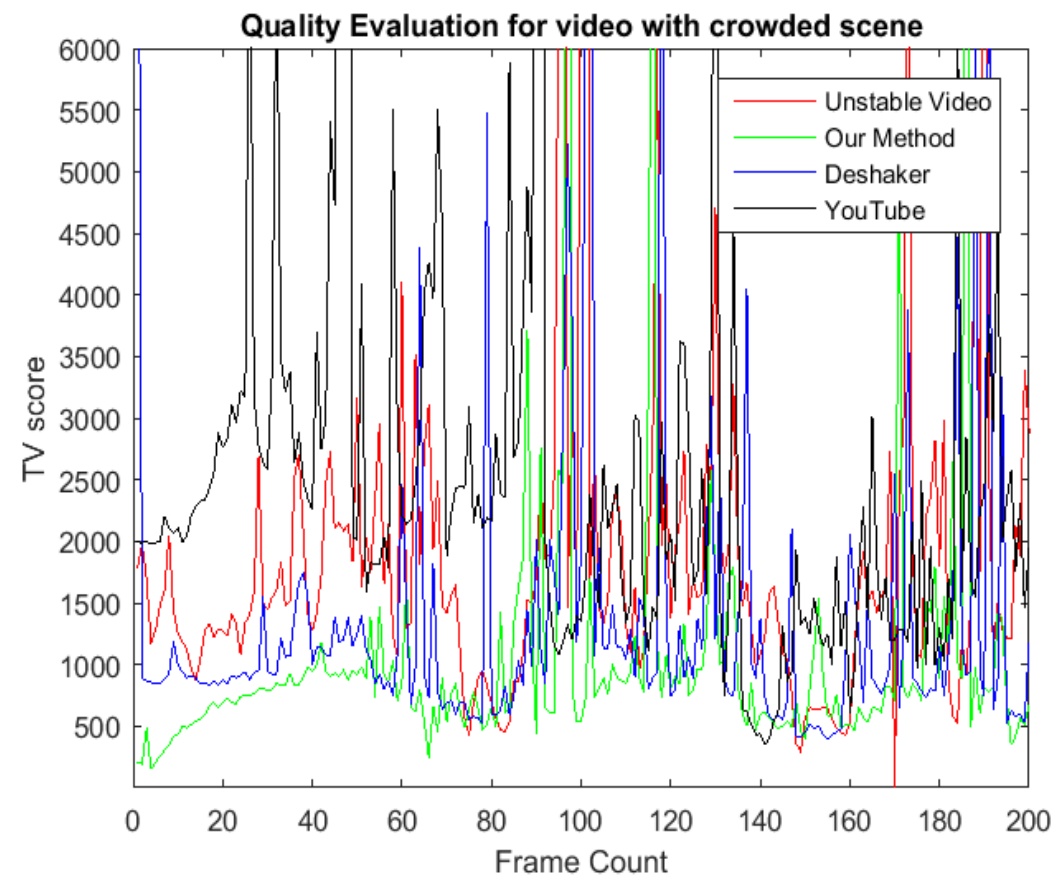

Figure 4.9 Jittering in the camera motion for unstable video with crowded scene, compared to the stabilised videos.

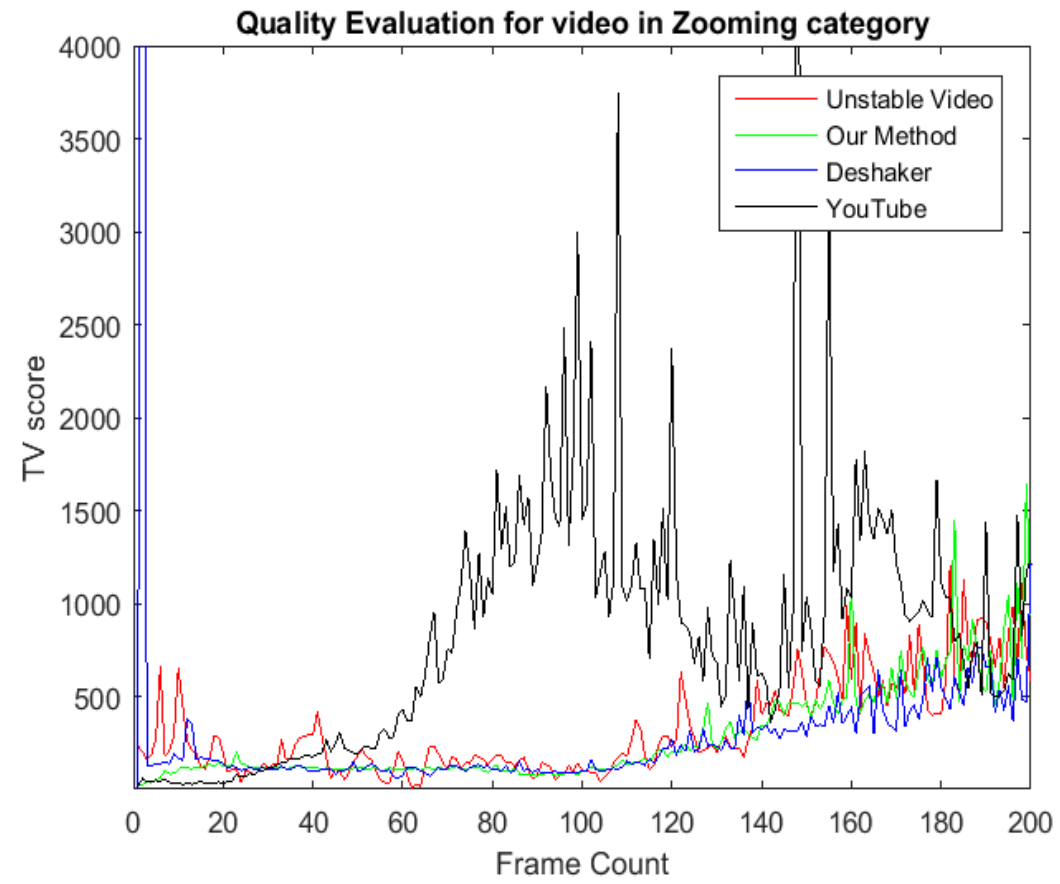

Figure 4.10 Jittering in the camera motion for unstable video with rapid zooming, compared to the stabilised videos. 


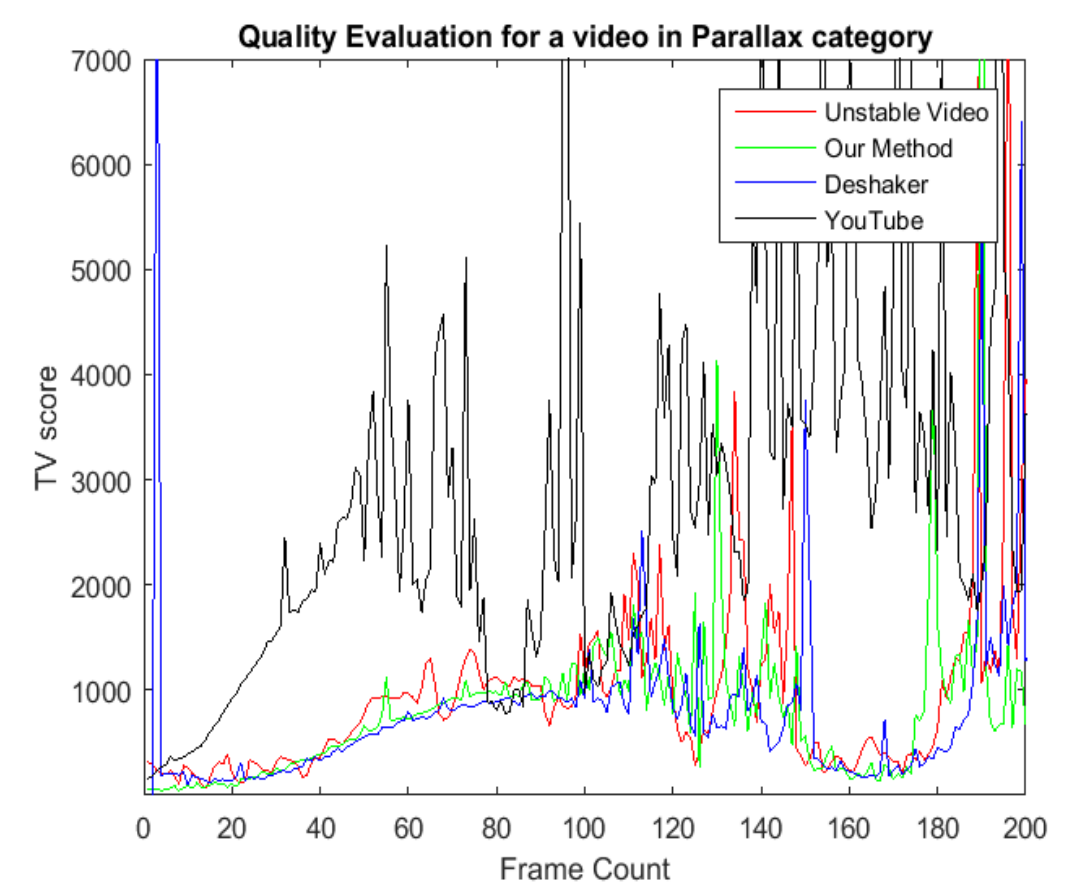

Figure 4.11 Jittering in the camera motion for unstable video with parallax, compared to the stabilised videos.

As it can be noticed in the above (Figure 4.7 to Figure 4.11), the plot in red has lot of jitteriness, which shows the undesirable camera shake in the unstable video. The plots in blue and green represents the camera path of stabilized videos using the Deshaker software and proposed video stabilization algorithm respectively. As it can be noticed, the plots of the stabilized video are smooth with less jittering compared to the unstable video. The plot in black represents the camera path of stabilized video using YouTube stabilizer. The black plot is not synchronizing anyway with input because the number of frames in the stabilized video using the YouTube editor is nearly half compared to the number of input frames. The reason is assumed that the YouTube compresses the video by estimating motion information and removing the very similar and slightly variant frames. 


\section{Conclusion}

The proposed 2-D motion estimation based video stabilization generated comparable results to commercial software and stabilization systems. A novel approach towards separately processing low feature count and blurry frames is one of the interesting findings. Most of the challenging consumer-level videos can be stabilized using this method. But, there are still some limitations that are discussed in the following.

The proposed novel quality evaluation helped in objectively estimating the jitteriness in the camera motion and also to compare various stabilization techniques. But this method assumes that the estimated camera path is the actual camera path of the video, which is not always true. The limitations are detailed in the following.

\subsection{Limitations of video stabilization method}

There are cases when there is sever occlusion or dominant foreground, then large portion of corresponding image features are on the foreground moving objects. Using RANSAC or MSAC to eliminate such outliers is not completely possible and results in false motion models and wobbling distortions as shown in Figure 5.1.

There are cases when there is sever rotation and blurring in the video frame and so even the minimum number of features cannot be detected. In such cases, the motion estimation can fail. We think that, for such severely affected frames, stabilization is unnecessary.

There are cases where the warping results in larger cropping of the frame when two or more challenges are severely present in the video. When there is sever occlusion added to rolling shitter effect, the proposed video stabilization technique resulted in excessive blank image after frame warping. 

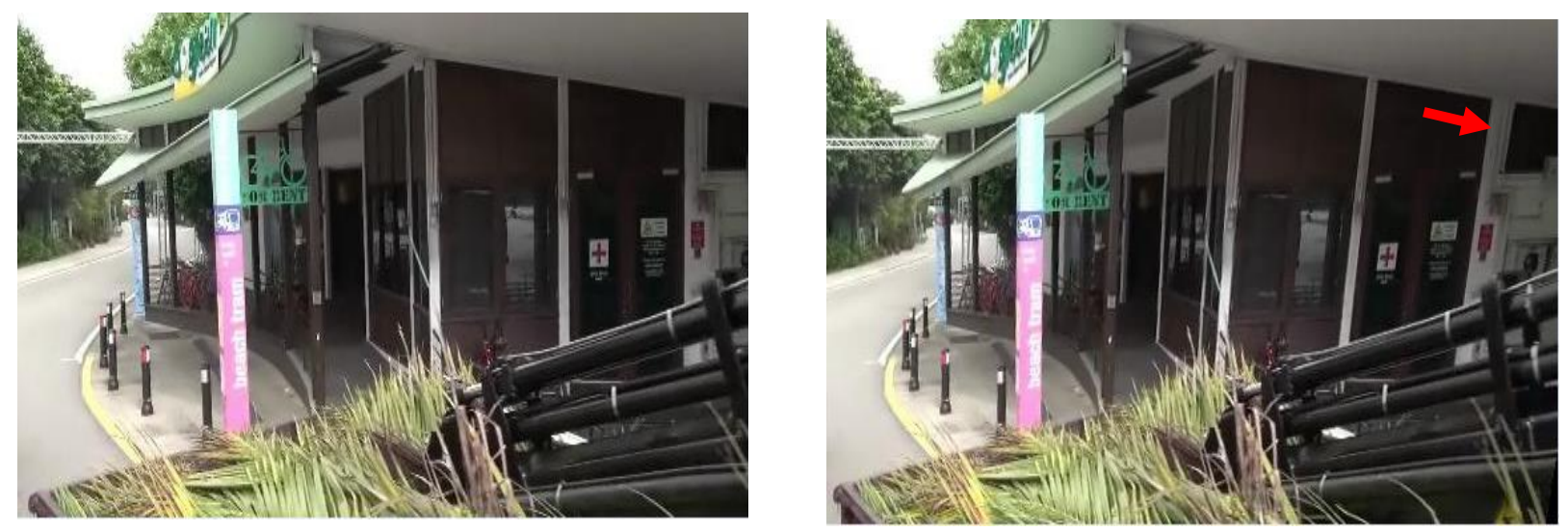

Figure 5.1 Wobble distortion in the stabilized video using proposed method due to sever occlusion in the foreground.

Also, this work do not process the visible blur artifacts contained in the stabilized video. While motion blur in original videos looks natural, it becomes a visible artifact in stabilized videos because it does not correspond to the compensated camera motion. [1, 54] have addressed this issue by replacing the blurry region with similar sharp regions in the nearby frames. So, our method can be combined with such techniques to generate non-blur images.

\subsection{Limitations of quality evaluation metric}

For the proposed quality evaluation metric, whenever a feature trajectory is lost, a new feature is introduced using K-Nearest Neighbor search. But the replaced feature may not represent the actual camera path; for it can be a feature from occlusion in the foreground. When foreground object features are less dominant compared to image features, it may not have effect on camera path estimation. But, when most of new features are from distracting foreground objects, the estimated camera path will be a false camera path. This can happen in videos with large occlusion in the foreground and videos with moving crowd. Sometimes the feature tracks can also fail when there is no rigid object in the scene. 

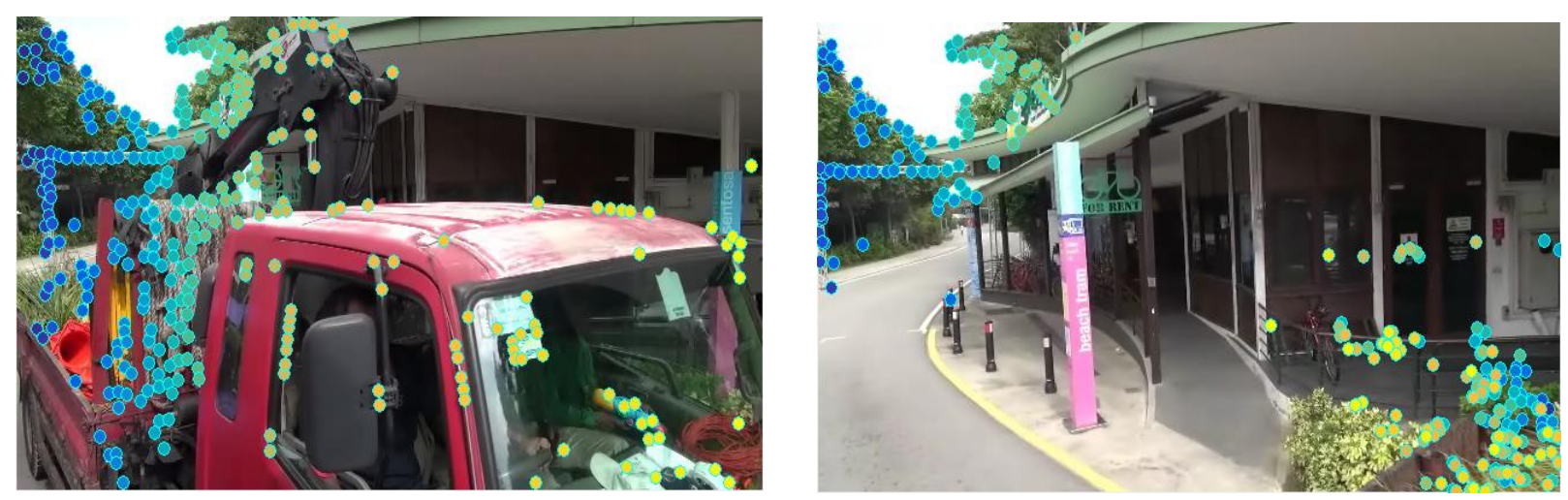

Figure 5.2 False camera path due to distracting foreground moving object.

Figure 5.2 shows two video frames from a video of total 327 frames. For the first 220 frames, the camera motion is absent and just the crane truck in the foreground is moving. In this case, the result of quality evaluation metric as shown in Figure 5.3 is a false camera jittering.

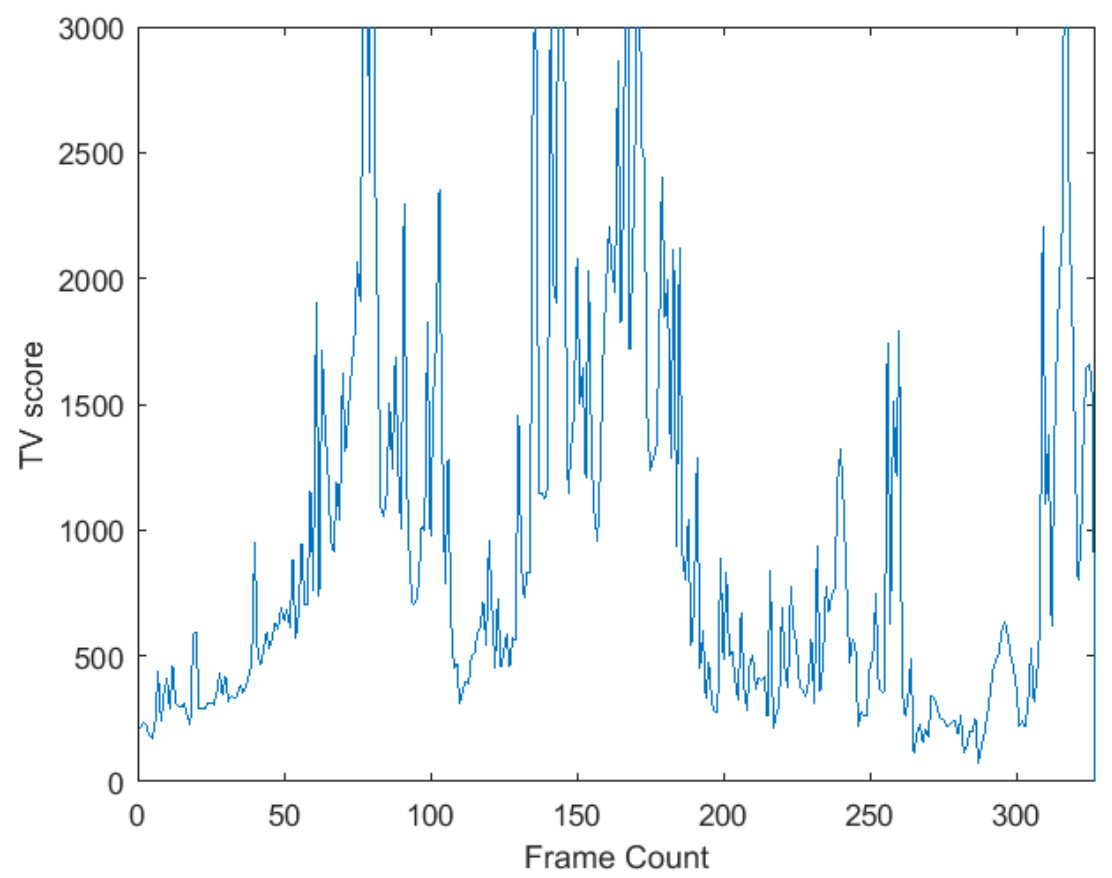

Figure 5.3 False camera jittering for a video with distractive foreground. 


\subsection{Future Research Directions}

This work proposes a 2-D motion estimation based video stabilization. As 2-D motion models are imprecise in the presence of large depth changes, 3-D motion estimation comes into picture. 3-D motion can be estimated by using depth sensor such as Kinect camera and can achieve high-quality appearance through exploring 3-D camera motions and scene depth. This can be a major research direction.

When video contains dominant foreground objects, the proposed video stabilization and quality evaluation both fails to distinguish the camera motion. These objects can consistently occupy more than half area of a frame and exist for a long time. So, advanced motion segmentation should be incorporated to work together with video stabilization or some user interactions [55] are also favoured to this problem.

Video blurring looks natural in original video but it becomes a visible artifact in stabilized videos because it does not correspond to the compensated camera motion. It can also severely influence the quality of feature matching or tracking. [1,54] have addressed this issue by replacing the blurry region with similar sharp regions in the nearby frames. So there are potentials that these two problems can be solved together.

It is also worth to explore the possibility to stabilize a video with the help of hardware devices (e.g., gyroscopes [11]). Nowadays, video stabilization methods are either purely based on software as a post processing method or based on hardware for real-time applications. But, the hardware equipment can be very expensive. So, it can be explored to design some hybrid approaches to combine the benefits from software and hardware. However, for real-time applications, we need to design a new path smoothing strategy because we can only look at previous camera paths with unknown future frames.

Video stabilization problem is wider and a complete solution to address all the challenges can be computationally intense. Motion compensation in video stabilization results in missing image areas in the output stable video. Most of the video stabilization techniques crop the outer edges of the video to produce a good to watch output and thus end up resulting smaller frame size stabilized video compared to the input unstable video. We propose an inpainting method to fill the missing area in a video frame by interpolating with similar patches in the neighboring frames. This 
approach generalizes the video inpainting problem to an image inpainting problem by looking through the temporal axis of the video Figure 5.4.
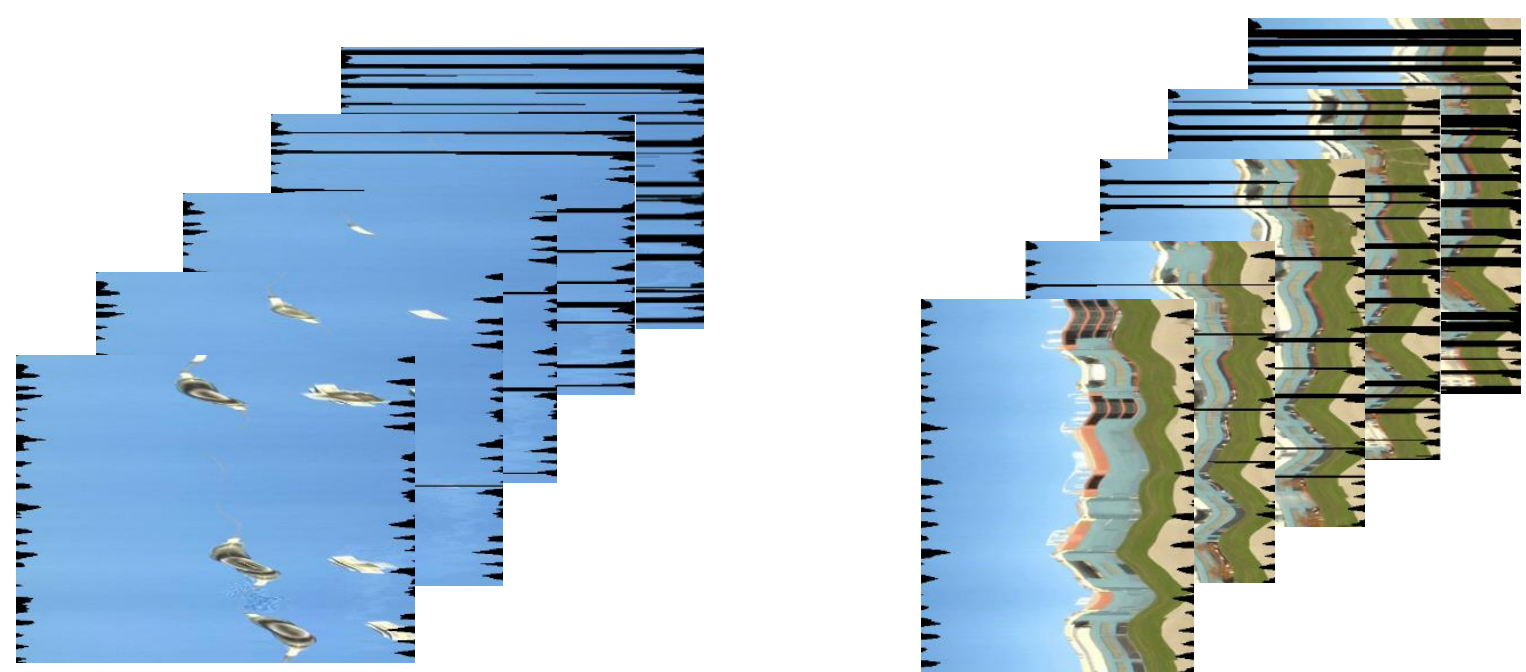

Figure 5.4 Temporal slices of a stabilized video. Black patches show the missing image regions due to motion compensation. Video inpainting can be addressed as image inpainting, by interpolating each temporal slice using similar patches from same slice or neighboring slices.

The result is a good output video, at the cost of computational complexity. Also, it requires the neighboring video frames to suffer from less motion to increase the chance of finding correct matches, which is not often guaranteed. There is a great potential to explore in this direction to restore the missing patches with better computation complexity. 


\section{Bibliography}

[1] Y. Matsushita, E. Ofek, W. Ge, X. Tang, and H.-Y. Shum. Full-frame video stabilization with motion inpainting. IEEE Trans. Pattern Anal. Mach. Intell., 28:1150-1163, 2006.

[2] F. Liu, M. Gleicher, H. Jin, and A. Agarwala. Content-preserving warps for 3D video stabilization. ACM Transactions on Graphics (TOG) (Proceedings of SIGGRAPH), 28, 2009.

[3] S. Liu, Y. Wang, L. Yuan, J. Bu, P. Tan, and J. Sun. Video stabilization with a depth camera. In IEEE Conference on Computer Vision and Pattern Recognition (CVPR), 2012.

[4] R. Hartley and A. Zisserman. Multiple View Geometry in Computer Vision. Cambridge University Press, New York, NY, USA, 2 edition, 2003.

[5] F. Liu, M. Gleicher, J. Wang, H. Jin, and A. Agarwala. Subspace video stabilization. ACM Transactions on Graphics (TOG), 30, 2011.

[6] M. Grundmann. Computational video: Post-processing methods for stabilization, retargeting and segmentation. Doctoral Thesis. Georgia Institute of Technology, 2013.

[7] S. Liu, L. Yuan, P. Tan, and J. Sun. Bundled camera paths for video stabilization. ACM Transactions on Graphics (TOG), 32(4), 2013.

[8] Z. Zhu and Zhigang Zhu and Guangyou Xu and Yudong Yang and Jesse S. Jin. Camera Stabilization Based on 2.5D Motion Estimation and Inertial Motion Filtering. IEEE International Conference on Intelligent Vehicles, 1998.

[9] A. Goldstein and R. Fattal. Video stabilization using epipolar geometry. ACM Transactions on Graphics (TOG), 2012.

[10] C. Morimoto and R. Chellappa. Evaluation of image stabilization algorithms. In IEEE International Conference on Acoustics, Speech and Signal Processing, pages 2789 - 2792, 1998. 
[11] A. Karpenko, D. E. Jacobs, J. Baek, and M. Levoy. Digital video stabilization and rolling shutter correction using gyroscopes. In Stanford Computer Science Tech Report CSTR 2011-03, 2011.

[12] S. Liu. Digital video stabilization. Doctoral Thesis. National University of Singapore, 2014.

[13] B.-Y. Chen, K.-Y. Lee, W.-T. Huang, and J.-S. Lin. Capturing intention-based full-frame video stabilization. Computer Graphics Forum (CGF), 27(7):1805-1814, 2008.

[14] M. L. Gleicher and F. Liu. Re-cinematography: Improving the camera dynamics of casual video. In ACM Multimedia, 2007.

[15] M. L. Gleicher and F. Liu. Re-cinematography: Improving the camerawork of casual video. ACM Transactions on Multimedia Computing, Communications, and Applications, 5(1), 2008.

[16] M. Grundmann, V. Kwatra, and I. Essa. Auto-directed video stabilization with robust 11 optimal camera paths. In IEEE Conference on Computer Vision and Pattern Recognition (CVPR), 2011.

[17] T. Igarashi, T. Moscovich, and J. F. Hughes. As-rigid-as-possible shape manipulation. ACM Transactions on Graphics (TOG) (Proceedings of SIGGRAPH), 24(3):1134-1141, 2005.

[18] S. Schaefer, T. McPhail, and J. Warren. Image deformation using moving least squares. ACM Transactions on Graphics (TOG) (Proceedings of SIGGRAPH), 25(3):533-540, 2006.

[19] C. Tomasi and R. Manduchi. Bilateral filtering for gray and color images. In IEEE International Conference on Computer Vision (ICCV), pages 839-846, 1998.

[20] I. N. Bronshtein and K. A. Semendyayev. Handbook of Mathematics. Springer-Verlag, 1997. 
[21] M. Irani. Multi-frame correspondence estimation using subspace constraints. Int. J. Comput. Vision, 48:173-194, July 2002.

[22] C. Tomasi and T. Kanade. Shape and motion from image streams under orthography: a factorization method. International Journal of Computer Vision (IJCV), 9(2):137-154, 1992.

[23] A. Goh and R. Vidal. Segmenting motions of different types by unsupervised manifold clustering. In IEEE Conference on Computer Vision and Pattern Recognition (CVPR), 2007.

[24] M. Irani. Multi-frame correspondence estimation using subspace constraints. International Journal of Computer Vision (IJCV), 48(3):173-194, 2002.

[25] G. Zhang, Z. Dong, J. Jia, T.-T. Wong, and H. Bao. Efficient non-consecutive feature tracking for structure-from-motion. In European Conference on Computer Vision (ECCV), 2010.

[26] G. Zhang, X. Qin, W. Hua, T.-T. Wong, P.-A. Heng, and H. Bao. Robust metric reconstruction from challenging video sequences. In IEEE Conference on Computer Vision and Pattern Recognition (CVPR), 2007.

[27] B. M. Smith, L. Zhang, H. Jin, and A. Agarwala. Light field video stabilization. In IEEE International Conference on Computer Vision (ICCV), 2009.

[28] T. Igarashi, T. Moscovich, and J. F. Hughes. As-rigid-as-possible shape manipulation. ACM Transactions on Graphics (TOG) (Proceedings of SIGGRAPH), 24(3):1134-1141, 2005.

[29] BEIER, T., AND NEELY, S. Feature-based image metamorphosis. In Computer Graphics (Proceedings of SIGGRAPH 92), 35-42, 1992.

[30] P. Torr and A. Zisserman. MLESAC: A new robust estimator with application to estimating image geometry. Computer Vision and Image Understanding, 78:138-156, 2000. 
[31] D.G. Lowe, "Object Recognition from Local Scale-Invariant Features," Proc. Seventh Int'1 Conf. Computer Vision, pp. 1150- 1157, 1999.

[32] D. Lowe, "Distinctive Image Features from Scale-Invariant Keypoints," Int'1 J. Computer Vision, vol. 2, no. 60, pp. 91-110, 2004.

[33] H. Bay, T. Tuytelaars, and L. Van Gool. Surf: Speeded up robust features. In European Conference on Computer Vision, May 2006.

[34] J. Wu, Z. Cui, V.S. Sheng, "A comparative study of SIFT and its variants" Measurement Science Review (2013).

[35] L. Juan and O. Gwun "A comparison of sift, PCA-sift and surf", Int. J. Image Process., vol. 3, no. 5, pp.143-152, 2009.

[36] J. H. Friedman, J. Bentely, and R. A. Finkel. An Algorithm for Finding Best Matches in Logarithmic Expected Time, ACM Transactions on Mathematical Software 3, 209, 1977.

[37] Naber, G.L., The Geometry of Minkowski Spacetime, Springer, New York, (1992).

[38] J. V. de Oliveira and W. Pedrycz, Advances in Fuzzy Clustering and Its Applications, 2007 :Wiley

[39] Güting, R.H., Behr, T., and Xu, J., 2010b. Efficient k-nearest neighbor search on moving object trajectories. The VLDB Journal, 19, 687-714.

[40] Elan Dubrofsky “Homography Estimation”. Masters Thesis, University of British Columbia, Canada, 2009. 
[41] Martin A. Fischler and Robert C. Bolles, "Random Sample Consensus: A Paradigm for Model Fitting with Applications to Image Analysis and Automated Cartography" in Comm. of the ACM 24, pp: 381-395 1981.

[42] R. Hartley. In defense of the eight-point algorithm. In IEEE Transactions on Pattern Analysis and Machine Intelligence, volume 19, pages 580-593, June 1997.

[43] J. J. Lee and G. Y. Kim. Robust estimation of camera homography using fuzzy RANSAC. In ICCSA '07: International Conference on Computational Science and Its Applications, 2007.

[44] Tordoff, B., Murray, D.W.: Guided sampling and consensus for motion estimation. In: Heyden, A., Sparr, G., Nielsen, M., Johansen, P. (eds.) ECCV 2002. LNCS, vol. 2350, pp. 82-98. Springer, Heidelberg (2002).

[45] S. Choi, T. Kim, and W. Yu, "Performance Evaluation of RANSAC Family," Proc. British Machine Vision Conf. (BMVC ’09), 2009.

[46] M. Rogers and J. Graham, "Robust Active Shape Model Search," Proc. European Conf. Computer Vision, pp. 517-530, 2002.

[47] P.H.S. Torr and D.W. Murray (1997). "The Development and Comparison of Robust Methods for Estimating the Fundamental Matrix". International Journal of Computer Vision 24 (3): 271300.

[48] E. Rosten and T. Drummond. Machine learning for high-speed corner detection. In Proc. 9th European Conference on Computer Vision (ECCV’06), Graz, May 2006.

[49] Harris, C. and Stephens, M. 1988. A combined corner and edge detector. In Fourth Alvey Vision Conference, Manchester, UK, pp. 147- 151. 
[50] SHI, J. AND TOMASI, C. 1994. Good features to track. In Proceedings of the IEEE Conference on Computer Vision and Pattern Recognition. 593-600.

[51] Bruce D. Lucas and Takeo Kanade. An Iterative Image Registration Technique with an Application to Stereo Vision. International Joint Conference on Artificial Intelligence, pages 674$679,1981$.

[52] Carlo Tomasi and Takeo Kanade. Detection and Tracking of Point Features. Carnegie Mellon University Technical Report CMU-CS-91-132, April 1991.

[53] L.I. Rudin, S. Osher, and E. Fatemi, "Nonlinear total variation based noise removal algorithms," Physica D, Vol. 60, pp. 259-268, 1992.

[54] CHO, S., WANG, J., AND LEE, S. Video deblurring for hand-held cameras using patchbased synthesis. ACM Trans. Graph. (Proc. of SIGGRAPH) 31, 4, 2012.

[55] J. Bai, A. Agarwala, M. Agrawala, and R. Ramamoorthi. User-assisted video stabilization. In Computer Graphics Forum(CGF), volume 33, pages 61-70, 2014. 